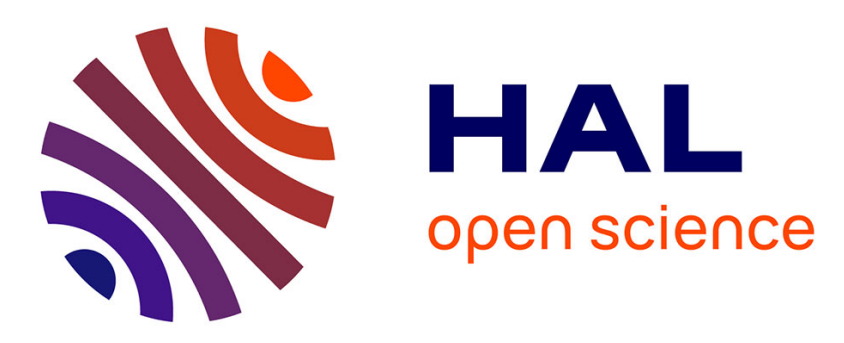

\title{
Three-dimensional Large Eddy Simulation of air entrainment under plunging breaking waves
}

Pierre Lubin, Stéphane Vincent, Stéphane Abadie, Jean-Paul Caltagirone

\section{To cite this version:}

Pierre Lubin, Stéphane Vincent, Stéphane Abadie, Jean-Paul Caltagirone. Three-dimensional Large Eddy Simulation of air entrainment under plunging breaking waves. Coastal Engineering, 2006, 53 (8), pp.631-655. 10.1016/j.coastaleng.2006.01.001 . hal-00294092

\section{HAL Id: hal-00294092 https://hal.science/hal-00294092}

Submitted on 8 Jul 2008

HAL is a multi-disciplinary open access archive for the deposit and dissemination of scientific research documents, whether they are published or not. The documents may come from teaching and research institutions in France or abroad, or from public or private research centers.
L'archive ouverte pluridisciplinaire HAL, est destinée au dépôt et à la diffusion de documents scientifiques de niveau recherche, publiés ou non, émanant des établissements d'enseignement et de recherche français ou étrangers, des laboratoires publics ou privés. 


\title{
Three-dimensional Large Eddy Simulation of
}

\section{air entrainment under plunging breaking}

\author{
waves
}

Pierre Lubin ${ }^{a, *}$, Stéphane Vincent ${ }^{a}$, Stéphane Abadie ${ }^{\mathrm{b}}$, Jean-Paul Caltagirone ${ }^{\mathrm{a}}$

a Transferts, Écoulements, Fluides, Énergétique (TREFLE), UMR CNRS 8508

École Nationale Supérieure de Chimie et de Physique de Bordeaux (ENSCPB)

Université Bordeaux 1

16 avenue Pey-Berland, 33607 Pessac Cedex, France.

${ }^{\mathrm{b}}$ Laboratoire des Sciences Appliquées au Génie Civil et Côtier (LaSAGeC ${ }^{2}$ )

Institut Supérieur Aquitain du Bâtiment et des Travaux Publics

Université de Pau et des Pays de l'Adour

Allée du parc Montaury, 64600 Anglet, France.

\begin{abstract}
The scope of this work is to present and discuss the results obtained from simulating three-dimensional plunging breaking waves by solving the Navier-Stokes equations, in air and water, coupled with a dynamic subgrid scale turbulence model (Large
\end{abstract}


Eddy Simulation, LES). An original numerical tool is used for the complete description of the plunging breaking processes including overturning, splash-up and the occurrence of air entrainment. The first part of the paper is devoted to the presentation and the validation of the numerical models and methods. Initial 3D conditions corresponding to unstable periodic sinusoidal waves of large amplitudes in periodic domains are then used to study further the ability of the numerical model to describe accurately the air entrainment occurring when waves break. The numerical results highlight the major role of this phenomenon in the energy dissipation process, through a high level of turbulence generation. The numerical model represents a substantial improvement in the numerical modelling of breaking waves since it includes the air entrainment process neglected in most previous existing models.

Key words: Navier-Stokes, Large Eddy Simulation, Two-phase flow, Plunging breaking waves, Splash-up, Air entrainment, Vortices

\section{Introduction}

Wave breaking is a very complex phenomenon, which is crucial to study as it plays an important role in sediment transport processes and in the trans-

* Corresponding author.

Email addresses: lubin@enscpb.fr (Pierre Lubin ), vincent@enscpb.fr

(Stéphane Vincent), stephane.abadie@univ-pau.fr (Stéphane Abadie), calta@enscpb.fr (Jean-Paul Caltagirone). 
fer of mass and momentum in coastal zones. During the last two decades, a number of important reviews have described and discussed in detail the general mechanisms involved in the breaking process (Peregrine, 1983) and the surf zone dynamics (Battjes, 1988; Svendsen and Putrevu, 1996), while Christensen et al. (2002) detailed the recent advances that have been made in the numerical modelling and the measurement techniques for the study of the surf zone. In this current study, plunging breakers are considered.

The jet-splash cycles, occurring several times in a single plunging breaker, are responsible for the generation of a sequence of large-scale coherent vortices. Some authors have highlighted the generation and the importance of the air entrainment during the wave breaking process. A review of this aspect of the research area has been presented by Battjes (1988). Among the most important works to consider, Miller (1976) investigated experimentally the internal velocity field, indicating the importance of what he called breaker vortices, the size and strength of which are a function of breaker shape. Some pictures from Miller (1976) indicate the formation of a large quantity of air bubbles during the jet-splash cycles and illustrate that the vortices in plunging breakers significantly affect the bottom flow. Nadaoka and Kondoh (1982) showed experimentally that the velocity field is characterized by the existence of very active turbulence associated with air entrainment, which is responsible for wave energy damping in the surf zone. In their measurements, it appears 
that the entrained air bubbles are contained mostly in the large structures and diffused towards the bottom due to the eddies. Lin and Hwung (1992) found from experimental measurements that the main mechanism driving the motion in the bubble region was the vortex system that was generated during the jetsplash cycles. Experiments showed that the eddies contained a large quantity of air bubbles which enhanced the upwelling of sediment. Chanson and Lee (1997) observed that the rate of energy dissipation was increased with the bubble penetration depth and with the characteristic length of the plunging jet shear flow. Chanson et al. (2002) studied experimentally the mechanisms of air bubble entrainment by plunging breakers. The results highlighted strong vertical motions induced by the rising air bubbles.

Recent progress in applied mathematics and computer architecture offer the possibility of developing numerical models for studying the breaking processes. The most direct method to investigate numerically the complexity of the flow in the surf zone is to solve the Navier-Stokes equations, coupled with a mathematical treatment for the free surface. The first and simplest way of solving the Navier-Stokes model is the Direct Numerical Simulation, with the assumption that the mesh grid size used to discretized the numerical domain is sufficiently refined to take into account all the length-scales of the flow, which is never the case in practice. The majority of the studies followed that method (Abadie et al., 1998; Chen et al., 1999; Iafrati et al., 2001; Abadie, 
2001; Guignard et al., 2001; Watanabe and Saeki, 2002; Lubin et al., 2003; Iafrati and Campana, 2003; Biausser et al., 2004; Song and Sirviente, 2004) and gave very promising results for the overall flow description, including the shoaling and the breaking of the waves. But the turbulence is not described or analyzed. A majority of these studies consider theoretical fluids, more viscous and lighter than water. Some recent research implemented the Reynolds Averaged Navier-Stokes (RANS) modelling of the surf zone (Lemos, 1992; Takikawa et al., 1997; Lin and Liu, 1998a,b; Bradford, 2000). These studies considerably improved in the understanding of the processes taking place in the surf zone but the turbulence levels at breaking were found to be overestimated. It should be noted that the work of Lin and Liu (1998a,b) and Bradford (2000) did not take the air entrainment into account. Except in the recent promising simulations shown by Lubin et al. (2003) and Biausser et al. (2004), all the previously cited work was two-dimensional simulations. Another very recent way of simulating turbulence in breaking waves is the Large Eddy Simulation (LES) method. Turbulence is taken into account in the NavierStokes equations thanks to a turbulence model for the subgrid scales of the flow. Zhao and Tanimoto (1998) first applied the LES method to breaking waves and showed very promising results compared with experimental measurements, considering a two-dimensional configuration. Mutsuda and Yasuda (2000) presented the first numerical results of three-dimensional Large Eddy 
Simulations of a plunging breaking wave, describing the air entrainment phenomenon. Christensen and Deigaard (2001) used a fully three-dimensional numerical tool based on the Navier-Stokes equations and studied spilling, weak and strong plunging breakers. Some very interesting visualizations and encouraging results describing the internal velocity field perturbed by threedimensional vortices were shown. Similar results were obtained by Watanabe and Saeki (1999) with three-dimensional numerical simulations of plunging breakers. Very recently, Hieu et al. (2004) studied two-dimensional breaking waves and Zhao et al. (2004) implemented a new multi-scale method where the two-dimensional flow structures were fully resolved with a $k-l$ RANS approach, while the three-dimensional turbulence interactions are modelled with a three-dimensional subgrid-scale eddy viscosity model. A good general agreement with experimental data was obtained, significantly improving the RANS results. Nevertheless, as the authors themselves stated, the air entrainment was not taken into account.

Few conclusions can be highlighted from this presentation of the previous studies. The experimental studies show that it is important to accurately describe the air entrainment process as it is responsible for a large amount of gas being entrapped and entrained in the water, which, in turn, plays a considerable role in the dissipation of the wave energy. Moreover, plunging breakers have the ability to entrain a large quantity of air at great depths, leading to long 
bubble residence time in the water column, which induces a large dissipation of energy (Chanson and Lee, 1997; Chanson et al., 2002). A considerable improvement in the numerical methods dedicated to the Navier-Stokes equations has been demonstrated, enabling the free surface and the general behavior of turbulent flow structures to be described with a very promising accuracy. Nevertheless, all the authors agree that solving the Navier-Stokes equations in an air/water configuration is a real challenge, due to the strong interface deformations and air entrainment phenomenon. Thus, the effect of air has not been studied yet in detail in most of the cited two- and three-dimensional simulations. The numerical methods are then a field of research requiring efforts and improvements in order to gain in accuracy and speed. The objective of our work is first to present new numerical methods for solving the two-phase flow Navier-Stokes equations in air/water configurations, including an up-to-date subgrid-scale model implemented in the numerical tool to take turbulence into account. Finally, we aim to describe the air entrainment and the internal velocity field under broken waves in three-dimensional configurations, in order to contribute to the understanding of the energy dissipation processes involved in the wave breaking. We hve chosen to study plunging breaking waves as their potential for air entrainment is much greater than the other breaker types (Cokelet, 1977; Chanson and Lee, 1997). Indeed, Miller (1976) experimentally measured the average bubble concentation in plunging and spilling breakers 
and indicated a larger bubble density presence in plunging breakers (about 31 $\%$ in the late stage compared to $19 \%$ for spilling breakers).

The paper is organized in three sections. In what follows, Sec. 2 details the numerical tool and the interface tracking technique. In this section, we also discuss the originality of the methods. Two test cases are described to illustrate the ability of the numerical methods to handle accurately with two-phase flows. The scope of Sec. 3 is to present detailed three-dimensional descriptions of the overturning motion and the general flow motion observed during the breaking of a wave. The resulting splash-up phenomenon is accurately analyzed. A considerable care is taken to describe the generation of large scale vortices, the air entrainment process, and its implications for the turbulence generation and the dissipation energy occurring during the three-dimensional plunging breaking process.

\section{Aquilon: a numerical tool for simulating 3D free surface flows}

The Computational Fluid Dynamic (CFD) library Aquilon, developed in the TREFLE laboratory, is based on a fixed Eulerian grid framework in order to avoid the numerical complexity of Lagrangian grids when dealing with interfacial or free surface flows. This modelling approach, which consists of solving the Navier-Stokes equations, involves the introduction of a new variable for 
repairing the different phases, the phase function $C$. It requires the extension of the momentum equations to an Eulerian two-phase flow modelling. In this section, attention will be paid to describe the physical model dedicated to free surface flows. The originality of the numerical methods is then detailed. The numerical tool is well suited to deal with strong interface deformations occurring during wave breaking, for example, and generally with turbulence modelling in the presence of a free surface. The method is able to calculate complex flows in complex geometries. The drawbacks are the numerical diffusion, induced by the numerical schemes used to discretize and solve the equations, and the considerable CPU time, which can be very time consuming when three-dimensional problems are tackled. Nevertheless, this method gives access to much reliable information about the velocity, acceleration and pressure fields under the broken waves.

\subsection{Eulerian formulation of the two-phase Navier-Stokes equations}

As detailed by Scardovelli and Zaleski (1999) in their work dedicated to Direct Numerical Simulation of two-phase flows on Eulerian grids, a single fluid formulation of the conservation equations is relevant to describe unsteady free surface flows. If we assume the flow to be incompressible and no phase change occurs, the equation system reads (Eqs. 1-2): 


$$
\begin{gathered}
\nabla \cdot \mathbf{u}=0 \\
\rho\left(\frac{\partial \mathbf{u}}{\partial t}+\mathbf{u} \cdot \nabla \mathbf{u}\right)=-\nabla p+\rho \mathbf{g}+\nabla \cdot\left(\mu+\mu_{t}\right)\left[\nabla \mathbf{u}+\nabla^{T} \mathbf{u}\right]+\sigma \kappa \mathbf{n}_{i} \delta_{i}
\end{gathered}
$$

and

$$
\frac{\partial C}{\partial t}+\mathbf{u} \cdot \nabla C=0
$$

where $\mathbf{u}$ is the velocity, $C$ the phase function, $t$ the time, $p$ the pressure, $\mathbf{g}$ the gravity vector, $\rho$ the density, $\mu$ the dynamic viscosity, $\mu_{t}$ the turbulent viscosity, $\sigma$ the surface tension coefficient, $\kappa$ the mean curvature of interface, $\mathbf{n}_{i}$ the unit normal to interface and $\delta_{i}$ a Dirac function indicating interface. If we examine the set of equations Eqs. 1 and 2, the main difference between the single fluid model and the standard Navier-Stokes equations are the introduction of a new advection equation on the phase function $C$ and the definition of an interfacial source term (see Eq. 13) in the momentum equations for taking into account surface tension effects. Moreover, the local densities and viscosities are functions of the fluid to which they are related.

Concerning the modelling of turbulence, Direct Numerical Simulations (DNS) can be carried out with Eqs. 1 and 2 by stating $\mu_{t}=0$ and choosing a grid and a time step fine enough to capture the Kolmogorov lengthscale of turbulence. For $\mu_{t} \neq 0$, Large Eddy Simulation has been demonstrated to be a very reliable 
tool for the unsteady simulation of numerous flows. Large scale turbulence is described by solving the flow equations, while the small scale turbulence, which is not resolved by the flow model, is taken into account through a subgrid scale model. As it is not possible to capture all the turbulent fluctuations with a reasonable numerical computation, the philosophy of Large Eddy Simulation is then to represent the dissipative effect of the small turbulent structures with a turbulent viscosity.

In the single fluid model (Eqs. 1-2), the phase function $C$ is equal to 1 in one fluid and 0 in the other. The interface is defined by $C=0.5$. The magnitude of the physical characteristics of the fluids depends on the local phase. They are defined according to $C$ in a discontinuous manner as:

$$
\begin{aligned}
& \rho=\rho_{1} \text { and } \mu=\mu_{1} \text { if } C \geq 0.5 \\
& \rho=\rho_{0} \text { and } \mu=\mu_{0} \text { if } C<0.5
\end{aligned}
$$

where $\rho_{0}, \rho_{1}, \mu_{0}$ and $\mu_{1}$ are the densities and viscosities of fluid 0 and 1 respectively. 


\subsection{Subgrid-scale model}

The Smagorinsky model is usually used in existing studies involving Large Eddy Simulation of wave breaking (Christensen and Deigaard, 2001), but it has been proved to be much too dissipative (Sagaut, 1998). In our study, the turbulent viscosity is calculated with the Mixed Scale model (Sagaut, 1998) that is much more efficient. We also implemented a selective function to check that the velocity field is locally turbulent and requires a subgrid scale model to be turned on.

This model exhibits a triple dependency on the large and small structures of the resolved field as a function of the cut-off length. The eddy viscosity $\mu_{t}$ is calculated as follows (Eq. 4):

$$
\mu_{t}(\mathbf{x}, t)=\rho C_{M} \bar{\Delta}^{1+\alpha}(|\bar{S}|)^{\frac{\alpha}{2}}\left(q_{c}^{2}(\mathbf{x}, t)\right)^{\frac{1-\alpha}{2}}
$$

where $\bar{S}$ is the resolved deformation rate tensor, $C_{M}$ is the model constant chosen as $C_{M}=0.06, \alpha$ is a parameter which value varies between 0 and 1. Generally, and in the following, $\alpha$ is taken to be equal to 0.5. The cut-off length of the filter $\bar{\Delta}$ is evaluated as follows: 


$$
\bar{\Delta}=(\Delta x \Delta y \Delta z)^{\frac{1}{3}}
$$

where $\Delta x, \Delta y$ and $\Delta z$ are the sizes of the mesh grids in the respective directions, and $x, y$ and $z$, respectively, are the longitudinal, lateral and horizontal coordinates. The quantity $q_{c}$ represents the kinetic energy of the test field extracted from the resolved velocity field through the application of a test filter associated to the cut-off lengthscale $\tilde{\bar{\Delta}}>\bar{\Delta}$. We choose $\tilde{\bar{\Delta}}=2 \bar{\Delta}$, because it is the value which is most used and seems to give the best results. This subgrid kinetic energy is supposed to be equal to the kinetic energy at cut-off $q_{c}^{2}$, evaluated in real space as (Eq. 6):

$$
q_{c}^{2}(\mathbf{x}, t)=\frac{1}{2} \mathbf{u}(\mathbf{x}, t)^{\prime} \mathbf{u}(\mathbf{x}, t)^{\prime}
$$

where the test field velocity $\left(\mathbf{u}^{\prime}\right)$ can be evaluated thanks to an explicit test filter applied to the resolved scales, noted (.) (Eq. 7):

$$
\left(\mathbf{u}^{\prime}\right)=\mathbf{u}-\widetilde{\mathbf{u}}
$$

This explicit discrete filtering operation is a linear combination of the neighboring values (Sagaut, 1998; Sagaut and Grohens, 1999). A three-dimensional 
test filter results from the tensorial product of the following mono-dimensional three-points filter (Eq. 8):

$$
\widetilde{f_{i}}=\frac{1}{6}\left(\bar{f}_{i+1}+4 \bar{f}_{i}+\bar{f}_{i-1}\right)
$$

This weighted average is obtained by applying the Simpson rule to compute the average of the resolved variable $\bar{f}$ over the control cell surrounding the $i^{\text {th }}$ point. This test field velocity represents the high frequency part of the resolved velocity field. The use of this model does not require any complementary wall model, as the Smagorinsky model does, because the eddy viscosity vanishes when the kinetic energy tends to zero at cut-off. Some work has been produced to improve the simulation results by better adapting the subgrid models to the local state of the flow. In order to improve the previously detailed subgrid-scale models, we use a sensor based on local information of the flow (David, 1993). This sensor, or selection function, is related to the local angular fluctuations of the vorticity $\omega$. All the details can be found in the book of Sagaut (1998). Our numerical model has been fully validated though numerous configurations, such as, for example, the interactions between a solitary wave and a submerged reef (Lubin, 2004; Lubin et al., 2004). 


\subsection{Discretization and solvers for the momentum equations}

The main difficulty concerning the numerical treatment of the Navier-Stokes equations (Eqs. 1) is the coupling between pressure and velocity and the fulfilment of the incompressibility condition. Following the work of Fortin and Glowinski (1982) on single phase incompressible flows, an augmented Lagrangian approach has been implemented in order to solve, at the same time, the momentum conservation and the mass balance through a minimization procedure (Vincent and Caltagirone, 1999, 2000). The augmented Lagrangian method (AL) consists in introducing a pseudo pressure equation:

$$
p^{n+1}=p^{n}-r \nabla \cdot \mathbf{u}^{n+1}
$$

and discretizing the momentum equation in time while replacing, at the same time, the implicit pressure by its expression given by Eq. 9 . If $\Delta t$ is defined as the time step and $n$ represents the numerical subscript corresponding to time $t=n \Delta t$, we obtain: 


$$
\left\{\begin{array}{l}
\rho\left(\frac{\mathbf{u}^{n+1}-\mathbf{u}^{n}}{\Delta t}+\mathbf{u}^{n} \cdot \nabla \mathbf{u}^{n+1}\right)-\nabla\left[r^{\prime} \nabla \cdot \mathbf{u}^{n+1}\right] \\
=-\nabla p^{n}+\rho \mathbf{g}+\nabla \cdot\left(\mu+\mu_{t}\right)\left[\nabla \mathbf{u}^{n+1}+\nabla^{T} \mathbf{u}^{n+1}\right]+\sigma \kappa \mathbf{n}_{i} \delta_{i} \\
p^{n+1}=p^{n}-r \nabla \cdot \mathbf{u}^{n+1}
\end{array}\right.
$$

In Eqs. 10, a specific AL term appears, expressed as $-\nabla\left[r^{\prime} \nabla \cdot \mathbf{u}^{n+1}\right]$, in which $r^{\prime}$ is a numerical convergence parameter used to act on the incompressibility constraint. This technique is commonly called the penalty method (Angot et al., 1999; Khadra et al., 2000). For example, when $r^{\prime}=0$, the numerical model (Eq. 10) does not take into account the mass balance, whereas, when $r^{\prime} \rightarrow+\infty$, only the divergence free property is imposed. Thus, typical values of $r^{\prime}$ ranging between 1 and 1000 enables the momentum conservation and the divergence free constraint to be solved at the same time. The key point of the AL approach is to discretize implicitly the momentum equations in order to verify the physical requirement of solving all the velocity components simultaneously, while verifying $\nabla \cdot \mathbf{u}^{n+1}=0$.

An Uzawa algorithm (Uzawa, 1958) is used to numerically solve the minimization or penalty problem associated to Eq. 10. Let $\epsilon$ be a numerical threshold controlling incompressibility, with $10^{-2} \leq \epsilon \leq 10^{-15}$, the Uzawa AL algorithm 
for a single phase flow is expressed as:

While $\left\|\nabla \cdot \mathbf{u}^{n+1}\right\|>\epsilon$,

$$
\text { solve }\left\{\begin{array}{l}
\rho\left(\frac{\mathbf{u}^{n+1}-\mathbf{u}^{n}}{\Delta t}+\mathbf{u}^{n} \cdot \nabla \mathbf{u}^{n+1}\right)-\nabla\left[r^{\prime} \nabla \cdot \mathbf{u}^{n+1}\right] \\
=-\nabla p^{n}+\rho \mathbf{g}+\nabla \cdot\left(\mu+\mu_{t}\right)\left[\nabla \mathbf{u}^{n+1}+\nabla^{T} \mathbf{u}^{n+1}\right]+\sigma \kappa \mathbf{n}_{i} \delta_{i} \\
p^{n+1}=p^{n}-r \nabla \cdot \mathbf{u}^{n+1}
\end{array}\right.
$$

with $p^{n+1}$ being a numerical Lagrangian term accumulating the explicit contribution of $\nabla \cdot \mathbf{u}^{n+1}$ to the incompressibility condition.

The multiphase flow version of algorithm (Eq. 11) requires the definition of a local penalty parameter in order to take into account the local variations of $\rho$ and $\mu$ due to the presence of a free surface (Vincent et al., 2004b). This approach is called the adaptative augmented Lagrangian (AAL) method and it implies the following definitions:

$$
\left\{\begin{array}{l}
r^{\prime}=K \max \left(\rho(t, \mathbf{M}) \frac{L_{0}^{2}}{t_{0}}, \rho(t, \mathbf{M}) u_{0} L_{0}, \rho(t, \mathbf{M}) \frac{L_{0}^{2}}{u_{0}} g, \frac{p_{0} L_{0}}{u_{0}}\right. \\
\left.\mu(t, \mathbf{M})+\mu_{t}(t, \mathbf{M}), \frac{\sigma}{u_{0}}\right) \\
r^{\prime}=r
\end{array}\right.
$$

where $K$ is a penalty constant ensuring the augmented Lagrangian term to 
be 2 to 3 orders of magnitude more than the larger term in the momentum equations. Practically, $K$ is chosen to be between $10^{2}$ and $10^{3}$. The vector coordinates $\mathbf{M}$ is defined as $(x, y, z)$ in a Cartesian framework. In expression Eq. 12, $\rho(t, \mathbf{M})$ and $\mu(t, \mathbf{M})$ are the local density and viscosity defined by Eq. 3 whereas $L_{0}, t_{0}, u_{0}$ and $p_{0}$ are characteristic lengthscale, time, velocity and pressure respectively.

To finish with the numerical solving of Eq. 1, a fixed staggered structured curvilinear grid is used to ensure an Eulerian representation of free surface flows. The space derivatives of the inertial term are discretized by a hybrid Upwind-Centered scheme of Patankar (1990) and the viscous term is approximated by a second order Centered scheme. The implicit discretization in time induces the building of a linear system which is solved thanks to an iterative BiCGSTAB II solver of VanDerVorst (1992). This method is preconditioned under a Modified and Incomplete LU (MILU) technique of Gustafsson (1978) to account for the strong disparities between terms in the linear system.

\subsection{Interface capturing method and surface tension discretization}

If the constitutive equations of the free surface flow (Eqs. 1-3) are examined, it can be observed that the distribution of the phase function $C$ plays a very important role in the model. Indeed, the cells cut by the interface are deduced 
from $C$, the physical characteristics of the flow are defined according to $C$ and the velocity field is highly dependent on the density and viscosity values. As a consequence, the solving of Eq. 2 is of major interest to ensure the consistency and accuracy of the numerical solutions.

In the past 10 years, much research has been dedicated to interface tracking on Eulerian grids: among those usually used most, we can cite the Volume Of Fluid method (VOF) of Hirt and Nichols (1981), improved for example by Gueyffier et al. (1999), the level set method of Osher and Sethian (1988) or the front tracking method of Unverdi and Tryggvason (1992). Liu (1999) gave a complete overview and discussion of the different numerical techniques that have been used for the interface tracking in numerical simulations of breaking waves, whereas Lubin (2004) detailed the most recent references. Instead of describing the free surface evolutions with the previous cited methods, which are based on interface reconstruction steps, we chose to solve directly the advection of $C$. Given that Eq. 2 is hyperbolic and $C$ is discontinuous, an explicit Total Variation Decreasing (TVD) Lax-Wendroff (LW) scheme of LeVeque (1992) is used to solve directly the interface evolutions without the reconstruction of $C$. When the small scale structures of interface are large compared to the grid size, the LW-TVD method is accurate and involves a controlled numerical diffusion across the interface on three grid points. The advantage of the LW-TVD approach is its easy programming in two- and three-dimensions and its low 
computational cost. Details of this numerical scheme and physical validations are provided in Vincent and Caltagirone (1999, 2000).

Since an Eulerian model has been chosen for the motion equations, a volume interpretation of the surface tension force $\mathbf{F}_{S T}=\sigma \kappa \mathbf{n}_{i} \delta_{i}$ must be proposed to take into account correctly this forcing in the cells cut by the interface. Our model is based on the volume reformulation of Brackbill et al. (1992) who introduced a continuous transition of length $h$ between the two phases, corresponding to an interface thickness. In its developments, the surface tension coefficient is assumed to be constant, the mean curvature is modelled by the divergence of the unit normal to the interface and the normal restricted to the interface is the gradient of the phase function $C$. In this approach, the thickness $h$ of the interface is in fact the numerical transition of $C$ between 0 and 1 across the contact surface between the fluids. Finally, the following Continuous Surface Force (CSF) is obtained:

$$
\mathbf{F}_{S T}=\sigma \nabla \cdot\left(\frac{\nabla C}{\|\nabla C\|}\right) \nabla C
$$

The convergence of model (9) to $\mathbf{F}_{S T}$, when $h \rightarrow 0$, has been demonstrated by Brackbill et al. (1992). The main drawback of spreading the interface on several grid cells is the introduction of numerical errors and the generation of spurious currents when studying steady state free surface flows involving 
capillary forces. These parasitic velocities were first reported by Lafaurie et al. (1994). Based on the Laplace number $L a=\sigma \rho R \mu^{2}$, Scardovelli and Zaleski (1999) estimated the limit beyond which the numerical simulations are impossible. The magnitude of $L a \simeq 10^{6}$ is practically measured in numerical developments. In the typical cases of solitary waves propagating on a water/air surface, as proposed in section (2.5.1), where a maximum curvature radius $R$ of the order of $10^{-3} \mathrm{~m}$ is observed, the Laplace number has a maximum of $8 \cdot 10^{4}$, which is less than the limit proposed by Scardovelli and Zaleski (1999). By this method, the parasitic currents will not affect interfacial flows and the choice of the CSF model is clever in the framework of solitary wave problems. Detailed methods for discretizing the surface tension force are given for example by Brackbill et al. (1992) and Vincent and Caltagirone (2000). In the following, we give the values 0 in the air and 1 in the water to the phase function $C$.

\subsection{Numerical validation}

When simulating two-phase flows, it is important to evaluate the general accuracy of the numerical methods and numerical schemes by checking the balance of mass and energy in the computing domain. The LW-TVD scheme and the surface tension modelling have been validated in numerous multiphase flow configurations (Vincent and Caltagirone, 2004; Vincent et al., 2004a). The nu- 
merical code has already been presented and proved to give accurate results for coastal applications (Lubin et al., 2003, 2004; Helluy et al., 2005). In order to present the physical meaning and the numerical behavior of our numerical model, we consider three academic problems: the solitary wave propagation in a constant water depth over a flat bottom, the rise of a three-dimensional spherical cap bubble in a stagnant liquid and the breaking of a dam on a wet bottom. Experimental measurements or analytical solutions are available to evaluate the accuracy and the consistency of the numerical simulations. The free-surface flows are representative of interface deformations, unsteadiness, mass conservation and surface wave propagation. It also enables the evaluation of the accuracy of the interface description thanks to the TVD numerical scheme, compared with the usual VOF-PLIC method (Liu, 1999). The test cases illustrate the capability of the AAL approach to deal with incompressible free surface flows in the presence of strong density and viscosity gradients.

2.5.1 A solitary wave propagating in a constant water depth over a flat bottom

Solitary waves are known for having some interesting properties: indeed, such a wave has a symmetrical form with a single hump and propagates with a uniform velocity without changing form. Thus, any solitary wave theory can be used to compute the initial kinematic properties and simulate the wave 
propagation in constant depths over horizontal beds in periodic domains. The precision of the simulation is assessed by comparing the free surface shapes and velocities with the theoretical values.

We use the $1^{\text {st }}$ order solitary wave theory for the initial wave shape and velocity distribution (Lee et al., 1982; Lubin and Lemonnier, 2004). We consider a two-dimensional solitary wave with a relative amplitude $\epsilon=H / d=0.11$ propagating in a constant water depth $d=0.3020 \mathrm{~m}$, so the amplitude is $H=$ $0.03322 \mathrm{~m}$ and the crest ordinate is located at $z=0.33522 \mathrm{~m}$. The initial wave celerity is $c=1.8134 \mathrm{~m} \cdot \mathrm{s}^{-1}$. The crest is located in the middle of the numerical domain at the beginning of the calculation. Periodic boundary conditions are imposed in the propagation direction, so the wave moving out of the domain on one side re-enters on the other side. All the calculations are made with the densities and the viscosities of air and water $\left(\rho_{a}=1.1768 \mathrm{~kg} \cdot \mathrm{m}^{-3}\right.$ and $\rho_{w}=$ $1000 \mathrm{~kg} \cdot \mathrm{m}^{-3}, \mu_{a}=1.85 \times 10^{-5} \mathrm{~kg} \cdot \mathrm{m}^{-1} \cdot \mathrm{s}^{-1}$ and $\left.\mu_{w}=1 \times 10^{-3} \mathrm{~kg} \cdot \mathrm{m}^{-1} \cdot \mathrm{s}^{-1}\right)$, and the related surface tension $\left(\sigma=0.075 \mathrm{~N} . \mathrm{m}^{-1}\right)$. The two-dimensional numerical domain is $10 \mathrm{~m}$ long and $0.6 \mathrm{~m}$ high. It is discretized into $300 \times 150$ regular Cartesian cells. We impose a free slip condition at the lower boundary and a free condition at the upper boundary. The time step is constant and equals $\Delta t=1 \times 10^{-2} \mathrm{~s}$, which gives $45 \mathrm{~s}$ of propagation simulated as we run 4500 iterations.

We consider the celerity of the initial wave, to estimate the theoretical distance 
it has to propagate during the time of the simulation and compare it with the final position of the wave crest. We want to verify carefully that the solitary wave maintains its original shape as it propagates. We then also calculate the differences between the theoretical and numerical crest ordinates. We also take the opportunity to compare the VOF-PLIC and TVD schemes with the influence of the use of the surface tension $\sigma$.

As summarized in the following tables (Tables 1 and 2), we report the numerical errors calculated with the results obtained at the $4500^{\text {th }}$ iteration. During the 4500 iterations, the waves must reach $81.603 \mathrm{~m}$, which is verified as the wave passes eight times by its initial position (Table 1). It is observed that the wave maintains its original shape as it propagates without major distortion. We also verify that the mass is preserved during the computation (Table 2). Indeed, the amplitude of the solitary wave is approximately constant. The initial crest ordinate and volume are respectively $z_{i n i}=0.33522 \mathrm{~m}$ and vol $_{i n i}=3.116 \mathrm{~m}^{2}$, with the theoretical abscissa of the final crest being $x_{\text {fin }}=81.603 \mathrm{~m}$.

Tables 1 and 2 should be here

The present numerical model gives very satisfactory results for this simple propagation problem. We then prove the ability of the numerical tool to conserve both the mass and energy along the simulations. In addition, the mod- 
elling of the surface tension is tested. We also compare the VOF-PLIC and TVD methods in the description of the free surface, in terms of interface locations.

\subsubsection{Rise of a three-dimensional spherical cap bubble in a stagnant liquid}

The situation of the test-case is relative to a three-dimensional fluid inclusion rising in another fluid. The inclusion and the surrounding fluid are initially at rest. Gravity induced buoyancy is the only force inducing the motion. This test-case deserves special attention for the final shape of the rising bubble and for the precise transient build-up of the bubble velocity exhibiting an overshoot before reaching its final asymptotic value.

To reproduce the overshoot and to get the correct terminal velocity, a numerical method has to accurately take into account buoyancy, viscous stresses and surface tension effects. This test-case is usually considered as a preliminary one for a numerical method (Lebaigue et al., 2004). It then allows us to prove the ability of the numerical model to reproduce the dynamics of a single rising bubble before considering a large quantity of entrained gas pockets as generally occurs in plunging breaking waves. A grid convergence analysis is also presented to test the sensitivity of our method.

We thus consider a three-dimensional bubble, initially at rest, which diameter 
is $2.10^{-2} \mathrm{~m}$. The initial parameters are those considered by Hnat and Buckmaster (1976); Blanco-Alvarez (1995); Lebaigue et al. (2004). We present in Fig. 1 the time evolution of the three-dimensional bubble as it rises. In Fig. 3, we successfully compare the equilibrium shape of the inclusion with the experimental results from Hnat and Buckmaster (1976).

Figure 1 should be here

Figure 3 should be here

In Fig. 2, we present a grid-sensitivity test. We compare our numerical results for three different mesh grid sizes with the results from Blanco-Alvarez (1995) for the nondimensional time evolution of the bubble velocity. It can be clearly seen that our numerical results converge towards the solution as we refine the mesh grid size. The key features of the process are quantitatively very well simulated.

Figure 2 should be here

This test-case proves that our numerical model is able to reproduce the complex dynamics of a rising bubble, as soon as ten or more grid points describe the bubble diameter. 


\subsubsection{Breaking of a dam on a wet bottom}

The interest in the dam-break problem is due to the existence of numerous experimental and theoretical studies which can be used to validate the Direct Numerical Simulation approach for solving air-water free surface flows. The usual configuration found in the literature is the breaking of a dam on a dry bottom. However, a more complex case, in terms of breaking and splashing occurrence, has been studied experimentally by Stansby et al. (1998): the breaking of a dam on a wet bottom. This situation is extremely interesting as it puts the stress on strong free surface deformations, turbulent flow behavior and dynamic interactions between air and water. The objective in this section is to tackle this dam-break case to evaluate and validate the numerical methods for a three-dimensional configuration. The present results are compared with those published by Stansby et al. (1998).

Figure 4 should be here

As presented in Fig. 4, we consider an idealized three-dimensional dam separating an upstream column of quiescent water of height $H$ and length $L$, from a downstream region of water of height $h$. With respect to the work of Stansby et al. (1998) and the dimensions of the experimental flume, the values of $H, L$ and $h$ are $0.1 m, 0.01 m$ and $0.6 m$, respectively. The computational domain is $1.2 \mathrm{~m}$ long, $0.2 \mathrm{~m}$ high and $0.4 \mathrm{~m}$ wide. The mesh is $600 \times 100 \times 10$ points. 
The time step is fixed equal to $2 \cdot 10^{-4} s$ to ensure a Courant-Friedrichs-Levy condition less than 1, necessary for the explicit advection of the free surface.

Water columns of constant depths are initially considered on both sides of the dam, the water being assumed to be at rest. At $t=0 \mathrm{~s}$, the dam is suddenly destroyed or removed. Then, the flow motion is driven by gravity. A shock wave, or bore, is developed on the downstream side, with the water pushing down from above acting somewhat like a piston being pushed downstream with an acceleration. A vertical jet (a mushroom-like jet, as named in Stansby et al. (1998)) then occurs. This phenomenon can be compared with what happens during the breaking of waves.

The free surface profiles are compared with those from Stansby et al. (1998) at the same instants (Figs. 5). The free surface profiles are taken from the middle section of the 3D numerical simulation results. A good agreement can be seen in the numerical results, in terms of jet occurrence amplitude and the behavior of the splash-ups.

Figure 5 should be here

In Fig. 5a, we can see that the splash rises as high as the one measured experimentally. The bore propagates at the same speed (Fig. 5b) with the same height and width. The differences observed may come from the fact that Stansby et al. (1998) drew their free surface profiles from blurred pictures 
showing a large amount of foam. The velocity fields of water and air regions are shown separately for easy and clear inspection in Fig. 6. It can be observed that the free surface is distorted due to the interactions with the large eddies in the air. A gas pocket has been observed to be entrapped and is advected in the water.

Figure 6 should be here

We thus show a very good agreement in both profiles and characteristic times for the simulation of the breaking of a dam on a wet bottom, compared with the experimental data. The numerical model gives very satisfactory results for this three-dimensional turbulent two-phase problem.

\section{Large Eddy Simulation of air entrainment in plunging breakers}

\subsection{Definition of the initial conditions and parameters}

We use initial conditions corresponding to unstable periodic sinusoidal waves of large amplitude, with the initial quantities being calculated from the linear theory. This somewhat artificial wave breaking case has already been documented in several previous studies and proved to be effective at simulating plunging breaking waves (Cokelet, 1979; Vinje and Brevig, 1981; Abadie et al., 1998; Chen et al., 1999; Abadie, 2001; Lubin et al., 2003; Song and Sirviente, 
2004). Such a wave cannot remain steady as the initial velocity field in water is not in equilibrium with the initial wave profile. This instability grows up quickly and leads to wave breaking. The overturning motion is controlled by only two parameters, the initial steepness, $H / L$, and the dispersion parameter, $d / L$. It makes this approach interesting as we are then able to study any breaker type by varying only these flow parameters. It is also a convenient configuration as we can study accurately the breaking phenomenon in a smaller numerical domain, as it is periodical in the flow direction, compared with simulations of shoaling waves breaking over a sloping beach which are very demanding in terms of grid mesh points. Nevertheless, the method of an unstable sinusoidal wave is an artificial way of leading a wave to the breaking process, compared with the usual shoaling process. Although it has already been shown that there is a certain similarity with what can be observed in real conditions, the method has to have some limitations somewhere. The main restriction in the description comes from the fact that the numerical domain is periodical in the flow direction. This configuration is thus similar to simulating an infinity of waves breaking at the same time, which does not happen in reality. Moreover, in nature, waves break where some other waves have broken. In our case, waves will break in a "turbulence free" area.

The main difference from the previous published studies is in the initial conditions or the assumptions employed. Indeed, many authors studied numeri- 
cally plunging breakers, with the same artificial initial conditions, under the assumption of an inviscid flow (Cokelet, 1979; Vinje and Brevig, 1981). Moreover, some published studies have been done with the Navier-Stokes equations (Abadie et al., 1998; Chen et al., 1999; Abadie, 2001; Song and Sirviente, 2004), but the density and viscosity ratios used in the simulations were not representative of realistic water waves. This is indeed a major difference with the present work where simulations are carried out with the viscosities and densities of air and water. Song and Sirviente (2004) proved numerically that taking surface tension into account has a considerable impact on the size of the jet ejection intensity and the air entrapment. They also highlighted the effect of artificially increased density and viscosity ratios on the ability of the wave to reach the breaking point and the higher rate of energy dissipation with less air entrainment. So, in the results presented before, the real density and viscosity ratios are used for all simulations. Taking into account the three-dimensional aspect of the phenomenon is another originality of our study, as very few three-dimensional numerical studies can be found in the literature (Watanabe and Saeki, 1999; Mutsuda and Yasuda, 2000; Christensen and Deigaard, 2001; Lubin et al., 2003, 2004).

Figure 7 should be here

The calculation domain is periodic in the wave propagation direction (one wavelength long), with a free slip boundary condition in the lower limit, and a 
free boundary condition in the upper limit. As a periodic condition is imposed in the flow direction, the wave moving out of the domain on the right side will re-enter on the left side (Fig. 7). The reference variables of the initial incident wave are the celerity $c\left(m . s^{-1}\right)$, the period $T(s)$, the wavelength $L(m)$, the depth $d(m)$, the waveheight $H(m)$ and the densities and viscosities of air and water $\left(\mathrm{kg} \cdot \mathrm{m}^{-3}\right.$ and $\left.\mathrm{kg} \cdot \mathrm{m}^{-1} \cdot \mathrm{s}^{-1}\right)$. The flow motion is characterized by the Reynolds number, $R e=\rho_{w} c L / \mu_{w}$, the density ratio, $\rho_{a} / \rho_{w}$, the viscosity ratio, $\mu_{a} / \mu_{w}$, the initial steepness, $H / L$, and the dispersion parameter, $d / L$. Usually, the last two dimensionless numbers are respectively representative of the non linearity degree of the wave and the influence of the bottom on oscillatory movement. In our specific case, $H / L$ mainly controls the instability growth speed or, in other words, the time separating the start of the computation and the breaking.

We recall that the viscosity and density ratios are $\mu_{a} / \mu_{w}=1.85 \times 10^{-2}$ and $\rho_{a} / \rho_{w}=1.1768 \times 10^{-3}$. At the initial time of the simulation, the water velocity field in the wave is obtained from the linear theory. The initial quantities are thus given by Eqs. 14: 


$$
\begin{aligned}
& \eta_{0}=\frac{H}{2} \cos k x+d, \quad p_{0}=\rho g\left(z_{\text {int }}-z\right) \\
& u_{0}=\frac{\omega H}{2} \frac{\cosh k z}{\sinh k d^{*}} \cos k x, \quad v_{0}=\frac{\omega H}{2} \frac{\sinh k z}{\sinh k d^{*}} \sin k x
\end{aligned}
$$

where $\eta_{0}$ is the initial free-surface shape, $x$ and $z$ are respectively the horizontal and vertical coordinates, $u_{0}$ and $v_{0}$ are the initial velocity components, $p_{0}$ is the initial pressure field, $z_{\text {int }}$ is the height of the interface, $k=2 \pi / L$ is the wave number and $\omega=2 \pi / T$ is the angular frequency. Three-dimensional simulations are initialized with the two-dimensional $(x, z)$ solution spread in the $y$-direction. To prevent our three-dimensional simulations from having any symmetry problems, the free surface is kept uniform in shape and we introduce in the velocity field the information of a variation of water depth $d^{*}=d+\epsilon$, where $\epsilon$ is a small linear perturbation quantity function of $y$. This initial velocity field leads to an unsymmetrical flow with respect to the long-shore axis. This preliminary study has been fully detailed by Lubin et al. (2003).

Grid convergence analysis is quite difficult and questionable for this kind of flow characterized with unsteady free-surface breaking. It should also be noted that we artificially generate the breaking of the wave, so we are not able to do comparisons with experimental data, except qualitatively. Some tests have 
been carried out to satisfy ourselves about the effect of the mesh grid size. We choose to run simulations with a regular Cartesian grid of $250 \times 100 \times 25$ points, giving a mesh grid resolution of $\Delta x=\Delta z=\Delta y=4 \cdot 10^{-4} \mathrm{~m}$. The time step is approximately $0.1 \mathrm{~ms}$. Keeping a constant mesh grid resolution, we verified that for any value of the wavelength $L$ greater than $10 \mathrm{~cm}$, the wave breaking process was not affected by capillary effects. Nevertheless, the mesh grid size is still coarse to resolve small bubbles, but sufficient to obtain good qualitative results for large entrained gas pockets. Additional numerical work is undertaken to improve subgrid scale models dedicated to two-phase flows (Labourasse et al., 2004).

We present the initial condition with $H / L=0.13$ and $d / L=0.17$, without the velocity field vectors, in order to see the water medium (Fig. 7). The interface is located at $C=0.5$. Note that the Navier-Stokes equations are solved in air and water.

\subsection{General flow description}

The initial conditions of unstable periodic sinusoidal waves of large amplitudes enable us to generate a wide range of breaker types, from weak plunging to collapsing breakers (Galvin, 1968). Table 3 shows the range of values for the initial steepness, $H / L$, and the dispersion parameter, $d / L$, that have been used 
in a preliminary two-dimensional study. All simulations have been carried out for a constant wavelength. It is usually admitted that there is a continuous spectrum of shapes from spilling to plunging, then to surging at the end of the array (Galvin, 1968). The subject of our work is not to study the transition between the regimes, but to visually classify the different types of wave breaking we obtained by varying the control parameters to give an idea of the behavior of the method.

Table 3 should be here

What we call a weak plunging breaker is the limit between the plunging breaker and the spilling breaker. A small jet is ejected from the crest of the breaking wave and impacts in the very upper part of the face of the wave. Spilling breaking waves involve a much more complex combination between the wave propagation and the vorticity generation based on perturbations appearing on the steepening face of the wave (Duncan et al., 1999). The grid refinement we use is not sufficient enough to be able to capture the mechanisms responsible for the generation of a "pure" spilling breaking wave as it is not the purpose of this study. When we indicate that a wave is not breaking, it has to be understood that we do not observe any jet ejection at all. A collapsing breaker occurs when the wave explodes more than it breaks in the conventional sense. The wave is so large and unstable that, as it steepens, no jet is ejected but the whole face splashes from its lower part. The breaking of the waves comes very 
rapidly in the case of a plunging breaker, while it occurs more slowly in the spilling case. We observed that the starting time is less than one wave period (the beginning of the breaking process is usually considered when the wave front is almost vertical). This was confirmed by the work of New et al. (1985).

In the following, we propose to detail some examples taken from Table 3 . The characteristics of the chosen three-dimensional simulations are summarized in Table 4. Calculations are carried out for a constant wavelength, so the initial waveheights, water depths and wave celerities increase, as does the initial Reynolds numbers, whereas the wave periods decrease. The case studied by Abadie et al. (1998) was the plunging wave from Vinje and Brevig (1981), characterized by $H / L=0.13$ and $d / L=0.13$. We chose to consider plunging breaking waves in shallow water. A precise description of the whole breaking process is carried out, including some characteristic phenomena such as splashups generation and entrainment of gas in the water.

Table 4 should be here

\subsubsection{Overturning process}

We present a first configuration carried out with $H / L=0.13$ and $d / L=0.13$ in order to compare the free surface profiles, velocity and acceleration fields, this case referring to the Boundary Integral Element Method simulations 
(BIEM) of Vinje and Brevig (1981). The Reynolds number of the flow is $R e \simeq 32400$, based on the definition given previously. Comparisons between our numerical results and those obtained by Vinje and Brevig (1981) have been shown to be in good agreement in terms of free surface profiles, velocity and Lagrangian fields (Lubin, 2004). The overturning motion is illustrated in Figs. 8, where the surface profiles shown have been chosen to correspond to those presented in Vinje and Brevig (1981). It can be clearly seen that the method is appropriate to describe plunging breaking waves.

At the initial time of the simulation, the water velocity field in the wave is calculated from Eqs. 14 and the air is at rest. The wave propagates towards the right side of the domain and the free-surface shape becomes more and more asymmetric, particularly in the region of the crest (Fig. 8, $t=0.17 \mathrm{Ts}$ ). An increase in the crest elevation occurs as the trough depth decreases. The front face of the crest steepens and becomes vertical.

Figure 8 should be here

A general good agreement can be observed between the present simulation and the results of Vinje and Brevig (1981), up to the time where the front face of the wave steepens (Fig. $8 t=0.30 T s$ ). As already discussed (Abadie et al., 1998; Chen et al., 1999; Grilli et al., 2004; Lubin, 2004), some differences are clearly seen between results taken from BIEM and Navier-Stokes 
simulations. The most striking one is that plunging jet profiles from Vinje and Brevig (1981) look like sharpened hooked blades, whereas the profiles shown in Figs. 8 look like rounded finger tips. The jet and crest of the wave look bigger, with a thicker aspect than the BIEM ones. At $t=0.17 \mathrm{~T} s$ (Fig. 8c), the height of the wave, predicted in the present simulation, is slightly higher than in Vinje and Brevig's results. They also show a jet formed at the top of the crest, projected straight forward along an horizontal axis, whereas the jet shown in Figs. 8c, which is formed in the upper half of the face of the steepened wave, has a curled aspect. The volume of gas entrapped by the jet seems to be smaller, even if the distance of the impact is approximately the same. In addition to size and shape considerations, the other discrepancy lies in the time evolution, with the present results showing a jet impacting earlier than the jet shown by Vinje and Brevig (1981). This has been observed to happen in some configurations studied and detailed by Lubin (2004). These differences may be attributed as due to the BIEM calculations, which are based on potential flow theory that ignores the effects of water viscosity. Indeed, Chen et al. (1999) proved that potential-flow computations, with the effects of surface tension and viscosity taken into account, lead to results comparable to those obtained with Navier-Stokes models. Chen et al. (1999) also point out that the jet ejection is affected by the frictional and inertial influences of the air surrounding the plunging tongue of water, which is absent 
in BIEM computations. Chen et al. (1999) obtained similar results to ours, with greater viscosity and density ratios $\left(\mu_{a} / \mu_{w}=0.4\right.$ and $\left.\rho_{a} / \rho_{w}=10^{-2}\right)$ than those we considered $\left(\mu_{a} / \mu_{w}=1.85 \times 10^{-2}\right.$ and $\left.\rho_{a} / \rho_{w}=1.1768 \times 10^{-3}\right)$. Sakai et al. (1986), Takikawa et al. (1997), Lin and Liu (1998b), Yasuda et al. (1999), Mutsuda and Yasuda (2000), Iafrati et al. (2001), Watanabe and Saeki (2002), Iafrati and Campana (2003) and Biausser et al. (2004) also showed the same kind of profiles. All these authors employed numerical tools for solving the Navier-Stokes equations with various schemes and methods used to handle the interface tracking.

Figure 9 should be here

The kinematics and dynamics of the overturning motion are well reproduced by the present model as the three following accepted features of this overturning motion can be observed: high velocities in the impinging jet, high accelerations under the curl and low accelerations on the rear of the wave.

Once the front face of the crest steepens and becomes vertical, a jet of liquid is about to be projected from the crest of the wave. Lubin (2004) presented a comparison of the velocity and acceleration fields, computed at the instant $t=0.28 T s$, once the front face of the wave is vertical. A good agreement was found between our numerical results and the BIEM computation of Vinje and Brevig (1981). At $t=0.28 T s$, Vinje and Brevig (1981) found that the max- 
imum acceleration magnitude is located on the face of the wave and is about $2.4 \mathrm{~g}$, compared with maximum acceleration magnitude of $2.3 \mathrm{~g}$ obtained in the present simulation. They also indicated that the horizontal velocities at the wave crest are slightly larger than the phase velocity. The initial wave celerity is $c=0.324 \mathrm{~m} . \mathrm{s}^{-1}$. The present computed maximum horizontal velocity is about $u=0.351 \mathrm{~m} \cdot \mathrm{s}^{-1}$.

Then the tongue of water thrown from the crest develops and free falls down forward, developing a characteristic overturning motion (Fig. 8). As depicted in Fig. 9, a very regular velocity field is predicted in both media. The initialization of velocity and pressure fields in the water domain (the air being at rest) is immediately followed by strong flow dynamics in the air. A depression is created in the air and a region of low acceleration is observed in the water, on the rear of the wave. On the contrary, a large amount of air is pushed forward by the moving wave and the jet of water (Fig. 9b). A vortex above the crest is generated and follows the wave during its motion.

The maximum acceleration is located on the under side of the overhanging jet and is directed out of the fluid, towards the air. The jet is about to impact the undisturbed surface. As expected, a high velocity region is found in the impinging jet (Fig. 9a). The plunging jet closes over the air to form a tube around which there is a considerable circulation. The jet entrains this pocket of gas and hits the forward face of the wave, forcing up a second jet, a splash-up. 
Different breaking configurations and behaviors are shown later on by varying the initial parameters. It is important to be able to describe accurately the splash-up mechanism as it is responsible for a large amount of gas of being entrapped and entrained in the water, which, in turn, plays a considerable role in the dissipation of the wave energy.

\subsubsection{Details of the splash-up mechanism}

Peregrine (1981) was the first to discuss splashes in waterfalls and breaking waves and then presented (Peregrine, 1983) three possible modes of splash-up generation:

- (a): the jet rebounds, the front face of the wave acting like a solid body

- (b): the jet penetrates and pushes up the water from the previously undisturbed water

- (c): the jet penetrates, pushes up the water from the front face of the wave and participates in the formation of the splash-up

The first hypothesis was shown to be very unlikely by some pictures (Peregrine, 1983). Abadie et al. (1998) tried to propose an answer. In their observations, subsequent to the early time of the jet impact, it appeared that the water came from a mixing between the initial impinging jet and the previously undisturbed forward face of the wave. Yasuda et al. (1999) used the VOF method and 
numerically computed movements of markers originating from the overturning jet to track their orbits to show that the mass supplied from the jet was confined almost within and around the main entrapped gas pocket. It seems very credible this is what happens in real conditions (Peregrine, 1983), as confirmed by Bonmarin (1989).

To discuss more precisely this point, we show three examples of splash-up generations presented in Figs. 11, 12 and 13. We use a "numerically" colored or marked water in our initial conditions, as shown in Fig. 10, which enable us to identify where the water in the splash-up comes from.

If we can judge the strength of the wave by the height of the splash-up it generates, we could say that the first one $(H / L=0.10, d / L=0.10)$ is the strongest, then the second one $(H / L=0.13, d / L=0.13)$, and the weakest one $(H / L=0.13, d / L=0.17)$ is the last presented.

In the present simulations, the jets are predicted to first rebound, whatever the position of the plunge point or the angle between the falling crest and the front face of the wave, with the undisturbed face acting as a solid wall. This confirms the experimental investigations of Jansen (1986) and Lin and Hwung (1992) who observed that the jet of water was almost totally reflected when it hit the front face of the breaking wave. We might have expected that feature in the last case (Figs. 13), where the tongue of water impacts closer to the 
crest than in the other cases, the rebounds would have been predominant and more water from the impacting jet would have been found in the splash-up. However this is not the case, as the splash-ups presented in the three cases exhibit the same general behavior: the water from the splash-ups originates exclusively from the plunging jets, at the very beginning of the process. In the three cases, the jet shapes can be compared to finger tips, as the falling tongues of water interact with the air escaping from the pockets about to be closed. This shape has certainly an influence on the fact that the upper part of the splash-up is formed with a layer of water coming from the initial jet. It can be clearly seen in Figs. 11, 12 and 13, that the splash-ups are made of water from both the impinging jets and the forward faces of the waves, following one of Peregrine's hypothesis. Moreover, the upper thin layers of water from the plunging crest are almost the same in proportions and do not increase in quantity as the splash-ups develop. The splash-ups are then generated by the large amount of water pushed by the plunging jets.

The results we present in Figs. 12 were obtained for the case $H / L=0.13$, $d / L=0.13$. This configuration has been studied by Abadie et al. (1998) with different viscosities in both media, to artificially reduce the Reynolds number, than those considered in this study. We can see, from his numerical results (Abadie et al., 1998; Lubin, 2004), that the shape of the falling tongue of water does not look like a rounded finger tip, but seems to be directed a bit 
inward, towards the pocket of air to be trapped. The consequence is that a thinner layer of water is rebounding with the splash-up.

As Chanson and Lee (1997) recall, the jet impact velocity and the angle between the plunging jet and the free surface of the receiving fluid are two dominant parameters for estimating the amount of entrained air and the sizes of entrained bubbles. From our observations, we can add that the position of the plunge point or the angle between the falling crest and the front face of the wave do not influence the composition of the splash-up, but rather the behavior of the subsequent flow. Chanson and Lee (1997) showed that the location of the plunging jet impact with the free surface was always above the still water level, which is confirmed by our numerical results.

The most striking point is the depth of penetration of the impinging jets, from strong to weak plunging breakers (Abadie et al., 1998). It is clearly observed (Figs. 11, 12 and 13) that, in the three cases, the jets do not penetrate very deeply. The tongues of water separate, one part of the liquid feeds the upper part of the splash-up, while the other goes around the main pocket of entrapped gas where a considerable topologically induced circulation takes place. This confirms Yasuda's observations and answers Peregrine's question concerning the three possible modes of splash-up generation.

Figure 10 should be here 
Figure 12 should be here

Figure 13 should be here

We can point out that it can be usually observed in the pictures that the falling crest is already a mixture of air and water, with the tip of the tongue being mainly composed of droplets. Miller (1976) and Bonmarin (1989) published some pictures confirming this point, the foam region of the falling jet exhibiting a round shape. We can conclude then that our numerical results are not so far from what could be observed in reality.

The splash-up grows in size and can rise higher than the original wave, as it is pushed by the plunging jet (Figs. 11 and 12). We show in Figs. 14 an example of the velocity field distribution in the splash-up. A large amount of liquid coming from the undisturbed liquid is pushed to develop the splash-up (Fig. 14a). A high ascending velocity component is located in this region. A large amount of vorticity is then generated and the flow becomes very violent and turbulent in water. Even if some of the momentum is due to the percussion of the impacting tongue of water, the high velocities usually observed at the bottom appear to be due to the spreading of the main rotating vortices more than the penetration of the free falling jet, depending on the water depth.

Figure 14 should be here 


\subsection{Vortices generation and air entrainment}

The jet reconnection with the forward face of the wave gives rise to two processes which have distinct life times and behaviors depending on the initial wave strength. On one hand, gas pockets are entrapped, generating a mixing of air and water and creating strong dynamics, sometimes spread towards the bottom, as a large amount of air is entrained in the water. The number and the size of these structures are important, because the energy dissipation process is linked to their behavior during the breaking of the wave. On the other hand, once rising to its highest level, the splash-up will generate several successive splash-ups, which are decreasing in size (Fig. 14b). It can sometimes rise higher than the initial waveheight. The successive rebounds causes more air entrainment and energy dissipation, as it will be detailed in the next section (3.5). Depending on the splash-up configuration, several vortices will be generated.

Zhang and Sunamura (1990) studied the conditions for the occurrence of vortices using two dimensionless quantities, the breaker-type index given by Galvin (1968) and the Reynolds number. Zhang and Sunamura (1990) showed that a condition for the occurrence of visible vortices should be that the Reynolds number of the flow would be greater than $1.2 \times 10^{4}$, which is below our Reynolds numbers (Table 4). They also classified the occurrence of the 
oblique and horizontal vortices, and subclassified the horizontal vortex into four types, depending on the developmental characteristics of the vortex:

- type A: formation of a horizontal vortex that will change to oblique vortices

- type B: formation of horizontal vortices that will develop in sequence

- type C: formation of a horizontal vortex that will disappear in a short time

- type D: formation of a horizontal vortex that will change rapidly to nonsystematic vortices

According to the conclusions of Zhang and Sunamura (1990), we can expect to see oblique vortices or horizontal vortices that will change to oblique vortices if we choose configurations close to spilling breakers or plunging breakers inducing sequences of propagating horizontal vortices. Thus, the behavior and the life time of the observed vortices depends on the three-dimensionality of the phenomenon (Peregrine, 1983; Lin and Hwung, 1992). Another subtype of horizontal vortices, absent from Zhang's classification, has been observed to occur in our simulations: the counter-rotative vortices. Bonmarin (1989) studied experimentally the shape evolution of a plunging crest after breaking has started. The resulting splash-up phenomenon is fully observed and detailed, including its part in the air-entrainment process. Some very interesting pictures show the co-rotative vortices and, more original, the generation of counter-rotative vortices or anti-clockwise revolving vortices. These have been observed to be very important in the breaking process. 
We summarize in Table 5 the types of vortices we obtained in a preliminary two-dimensional numerical study, depending on the values of the initial steepnesses, $H / L$, and dispersion parameters, $d / L$. We collected this information to have an idea of what we observe from our numerical experiments. These are only qualitative and preliminary descriptions that enable us to choose some cases to study and illustrate more precisely the air entrainment process.

\section{Table 5 should be here}

We can anyway give some preliminary analysis. We can see that the type of vortices is clearly related to the size of the plunging jet. If we assume that the strength of the wave is connected to the plunging jet size, so the intensity of the impact will increase as the plunging jet size gets larger. It can be seen from Figs. 11, 12 and 13 that large plunging tongues of water generate large and spectacular splash-ups, this process being related to the type and number of vortices which will be observed. This confirms previous studies (Yasuda et al., 1997, 1999) which identified the jet size as being clearly linked to the characteristic values of the resultant large scale eddies and entrained air bubbles. We can conclude from Table 5 that small plunging jets will generate only co-rotative vortices, as the splash-ups do not rise very high but are responsible for a cycle of rebounds entrapping co-rotating gas pockets (Fig. 15). So we can see that counter-rotative vortices start to appear when the amplitude becomes larger, and thus the bigger the plunging crest is. The occurrence 
of the counter-rotative vortices coincide with large plunging breakers. These structures were observed by Bonmarin (1989) in the case where the splash-up rises high and expands into a mushroom-like shape, one part generating a new splash-up in the flow direction and the other part falling back on the initial plunging tongue of water. Once the initial splash-up falls down backwards on the initial impinging jet, a dipole is created, consisting in these two counterrotative vortices (Fig. 14). These two rotating structures match together to form a pair with a high velocity between them, directed towards the bottom of the wave. The flow between the two counter-rotative vortices, resulting from the impact of the projected tongue of water, is fed by the impacting jet. These vortices are observed to twist along the y-axis. These two vortices dissipate a large amount of the wave energy, due to the high quantity of air entrapped to be entrained towards the bottom and to their high speed of rotation. These structures transport all the momentum and vorticity to the bottom.

To illustrate the different behaviors of the dipoles of counter-rotative vortices we observed, we describe three examples of three-dimensional plunging breakers (Figs. 16, 19 and 20), simulated with the characteristics summarized in Table 4.

In the first example (Figs. 16), we can see that the main entrapped gas pockets are rising in the middle of the domain (Fig. 17). The two counter-rotative vortices are distorted and separating as one of them is stretching and rising to 
the surface, due to the buoyancy effect. In Figs. 17, we present three sections showing the velocity field in both air and water media and the main gas pocket rising to the free surface. Once it pierced the free surface (Fig. 18a), the velocity vectors in the air indicate the violent spray of gas ejected from the rising pocket (Fig. 18b).

Figure 16 should be here

Figure 17 should be here

Figure 18 should be here

Some of the co-rotative vortices can move forward and down into the interior of the wave, like a propagating bore, corresponding to the subtypes A or B from Zhang and Sunamura (1990) (Figs. 16). In some configurations, the co-rotative vortices were entrained to the bottom, or dislocated and brought to the free surface very violently, corresponding to the subtypes C or D from Zhang and Sunamura (1990) (Figs. 19 and 20). We can sometimes see coherent rotating structures and sometimes very chaotic ones: the more violent the plunging breaker is, the shorter the life time of the pockets of gas. We present in Figs. 19 and 20 the two other examples of three-dimensional Large Eddy Simulations giving an excellent description of the violent and spectacular behavior of two plunging breakers. 
Figure 19 should be here

Figure 20 should be here

When the jet is projected from the steepening crest of the wave, a first vortex is created, a pocket of air is entrapped and put into rotation due to the high circulation of water surrounding it (Figs. 16a, 19a and 20a). A large amount of gas is entrained in the water. The air resists, as it is enveloped by the tongue of water, and induces a high shear rate at the free-surface. The flow becomes very turbulent and violent in both media. We can then see that we obtained a high splash-up generating counter-rotative vortices, as observed by Bonmarin (1989), and co-rotative vortices, as observed by Miller (1976) or Sakai et al. (1986). After the jet touches down the forward face of the wave, a series of splash-ups induces some new vortices in front of the main one previously generated (Figs. 16b, 19b and 20b). This phenomenon appears to repeat (Miller, 1976; Bonmarin, 1989) and decrease in size as it develops. In Figs. 19 and 20, large splash-ups, rising higher than the initial waveheights, form a pair of counter-rotative vortices and large co-rotating vortices in front of these structures.

The entrapped gas pockets can have very various sizes. The major large vortices are firstly two-dimensional on average with an axis parallel to the wave crest, and then they break up into three-dimensional flow structures. The 
length scales of these large structures can be approximately from a third to a half of the whole depth (Fig. 19c and 20c). They sometimes reach the same size as the water depth as they are rotating and stretching (Fig. 20d). The speed of propagation of these vortices is observed to be smaller than the initial wave celerity. Some of the structures are forced to rise and explode through the free surface due to the rapid compression of the entrained pockets of air (Miller, 1976). We could see the gas pockets rising to the free surface and the air escaping (Figs. 18, $19 \mathrm{f}$ and 20f). Mutsuda and Yasuda (2000) described a similar behavior in the case of a plunging breaking solitary wave.

The behavior, the quantities and the sizes of the vortices generated depend on their trajectories and the strength of the initial wave. We present in Figs. 21a the trajectory of the first major pockets of air, which are entrapped by the plunging jets. The trajectory of the next pocket of air, which is entrapped by the splash-up, is shown in Fig. 21b. The trajectory is represented as the variation of normalized air pocket height $(z / L)$ with the normalized distance $(x / L)$, in order to compare the results from the three test cases. When $(z / L)=1$, the gas pocket has reached the free surface. This has to be compared with Figs. 6 and 7 from Miller (1976) who presented the trajectories of vortex centers relative to the crest and the rate of expansion of the first three successive vortices for plunging breakers. 
These observations have to be related to the sizes of the structures, as we visually estimated the average location of the core of each main pocket. We do not take into account the expansion and the stretching of the structures, as we are only interested in describing the general entrainment process.

From the three lines plotted in both Figs. 21a and 21b, the general behavior detailed previously is confirmed. In the case of the main tube of air, once it is generated, it is entrained in the water, then it rises very rapidly to the free surface. The structures are found to propagate less than half a wavelength in distance. The depth of penetration is found to be more than half of the initial water depth.

In the case of the first co-rotative spinning pocket of air, one of them is shown to be rising before being entrained in the water. This is due to the fact that a large amount of water is pushed by the plunging jet to develop a high splashup (Fig. 16a). These pockets of gas are seen to stay near the free surface and do not propagate along a long distance.

Even if the wave breaking process seems visually more violent in the $H / L=0.10$, $d / L=0.10$ and $H / L=0.13, d / L=0.13$ configurations than the $H / L=0.13$, $d / L=0.17$ one, the general behavior is found to be very similar in the three cases.

Figure 21 should be here 
Experimental data do not allow us to present a direct comparison with our numerical results, but our general descriptions are in a good agreement with the pictures and the conclusions presented by Miller (1976) concerning the behavior of the gas pockets.

\subsection{Turbulence generation}

The two main mechanisms for the production of turbulence are the topologically induced vorticity and the type of flow generated by the impacting jet on the forward face of the wave. As our study deals with a single breaking wave, the resulting flow is unstationary. Thus, it is not possible to calculate any mean quantity and, therefore, any fluctuating value, with respect to the time. This implies that we cannot have any information about the turbulent

kinetic energy $k$, which is defined as $k=\frac{1}{2}\left(\overline{u^{\prime 2}}+\overline{v^{\prime 2}}+\overline{{w^{\prime 2}}^{2}}\right)$, where $\overline{u^{\prime 2}}, \overline{v^{\prime 2}}$ and $\overline{w^{\prime 2}}$ are the mean of the squared fluctuating Cartesian component of the velocity field. Christensen and Deigaard (2001) averaged the velocity field over the transverse direction as an alternative method to the ensemble-averaging method. This is only possible in the case where the extension of the transverse direction is sufficiently large to obtain stable and reliable statistics. We chose not to follow this method and concentrated our work on the description of the larger-scale structures and the general behavior of the flow, as some numerical tests would be needed to ensure that the mean values are not influenced by a 
too narrow numerical domain.

We then used a more direct method based on a visual investigation of the turbulent viscosity to study the behavior of the flow. Figs. 22 show the time evolution of the calculated turbulent viscosity $\mu_{t}$ in both air and water media. The eddy viscosity can be used as an indicator for locating small scale turbulent regions, as the subgrid-scale models used are supposed to vanish if the flow is not fully turbulent.

We can then see that the turbulent viscosity is mainly located where the large scale structures are. The maximum values of turbulent viscosity are observed in the regions of strong shear stress. These maxima correspond to some spotted areas. Thus, the successive impacting splash-ups generate higher shear stress during rebounds compared to penetrations (Figs. 22a, b and c). This is why the co-rotative vortices do not propagate very deeply (upper half of the water depth). These spinning structures produce high turbulent viscosity values. They explode to the surface very quickly, after propagating along less than half the wavelength. It is a strong plunging breaker and the main part of the energy of the initial wave is dissipated at the impingement. We can see that turbulence is spread straight to the bottom by the two counter-vortices generated by the violent impact of the initial impinging jet (Figs. 22d to h). It is generally accepted that the energy dissipated during the breaking process is first converted into turbulent kinetic energy of organized vortices before being 
dissipated into small-scale turbulence.

Figure 22 should be here

Except in the initial impinging region, the turbulence remains located near the free-surface, where the co-rotative vortices propagate. If we pay attention to the propagating bore, it can be observed that turbulence is still active, even when the large structures have disappeared. Some high values in magnitude of the turbulent viscosity can be seen (Figs. $22 \mathrm{~g}$ and $\mathrm{h}$ ), showing the interactions between some volumes of fluid put into rotation by the large spinning pockets of gas. It has been seen that the behavior of the flow field was highly threedimensional, as expected. These observations are all consistent with the results found in literature. Nadaoka and Kondoh (1982) showed that the existence of intensive turbulence is associated with entrained air bubbles. Moreover, experimental results suggest that the turbulence is generated in the upper part of the water and then reaches the bottom. Mutsuda and Yasuda (2000) presented similar conclusions, as high tubulence intensities were calculated in areas of strong air-water mixing.

It can also be observed in Figs. 22 that some turbulence is spreading towards the bottom after the propagation of the co-rotative vortices. Zhang and Sunamura (1990) indicated that the occurrence of spilling breakers provides a necessary condition for the formation of oblique vortices, but showed 
that these particular eddies can be found where a bore propagates even if a plunging breaker occurs. This confirms the observations of Sakai et al. (1986) and Nadaoka et al. (1989) who identified the generation of these fully threedimensional structures. It is not possible to conclude with only the numerical simulations presented that we are able to observe these coherent structures. We can only say, after a careful investigation of the velocity field, that some chaotic motion is spreading towards the bottom.

If we look at the velocity field in Figs. $17 \mathrm{a}$ and $17 \mathrm{~b}$, we can see that a large volume of water is put into rotation. This eddy is as large as the water depth. Moreover, the main flow is directed towards the shoreline, which implies that the sediment would be entrained in this direction. This confirms that plunging breaking waves are responsible for a large amount of sediment that is suspended and for the formation of sandbars, whereas spilling breaking waves are responsible for the erosion of these sandbars.

We investigated the impact of Large Eddy Simulation compared with results obtained from Direct Numerical Simulations (Lubin et al., 2003). We have first checked that no difference appeared before the impact of the jet, as expected. Being a positive quantity, the turbulent viscosity is only dissipative, as it is supposed to reproduce the effects of turbulence. The coupling with a subgrid scale model is expected to lead then to some foreseeable results. For example, the interface could be slightly smothered, with all the small perturbations 
near the free surface being erased, or the flow turbulent motion could be damped more quickly. However, surprisingly, the interface appears to be much more vigorously agitated, compared with the Direct Numerical Simulation. The main difference is found in the behavior of the co- and counter-rotative vortices generated in the flow. While in the Direct Numerical Simulation, we could see a turbulent flow composed of large spinning and propagating parallel tubes of gas finally exploding to the surface after some time, in the Large Eddy Simulation we can see a turbulent "chaotic" flow composed of gas pockets concentrated in the middle of the numerical domain. The gas pockets disappear rapidly but put volumes of water into rotation. The broken wave looks more agitated, fluctuated and disordered, but the flow still looks like it develops into a propagating turbulent bore.

Unfortunately, it can be seen in our numerical results that no foam is observed, as the number of grid points is not sufficient enough to take into account the dislocation of the pockets of gas into small bubbles. We presented in section (2.5.2) that the description of a single rising bubble is already a very complex case and demanding in terms of mesh grid refinement. With past two-dimensional studies, we could check that the finer the grid was, the more pieces were produced from the entrained gas pockets, torn apart during the breaking of the wave. As pointed out by Lamarre and Melville (1991), the energy dissipation is dependent on this process. A parallel version of the 
numerical tool is under validation and will soon be used to simulate the flow with a larger number of grid points.

\subsection{Energy dissipation}

The wave energy dissipation process has been investigated. According to Eqs. 15, the kinetic energy, the potential energy and the total energy are:

$$
\begin{aligned}
& E_{k}=\frac{1}{2} \iint \rho u^{2} d x d y \\
& E_{p}=\iint \rho z d x d y \\
& E_{t}=E_{k}+E_{p}
\end{aligned}
$$

The integrations are calculated over the whole domain, in the liquid part $(C \neq 0)$. We take the bottom $z=0 m$ as the reference level for the calculation of the potential energy. We also define zero potential energy corresponding to a non-perturbed surface, and we normalize the values of the energies by the respective initial values.

We plot in Fig. 23 the time evolution of the normalized values (by each respective initial values) of the kinetic, potential and total energies, for the case where $H / L=0.10, d / L=0.10$. The results we show are in good general agree- 
ment with the work of Chen et al. (1999), as we find that the time evolution of the calculated energies present three distinct regimes before, during and after the breaking of the wave.

Figure 23 should be here

Before the ejection of the jet $(t<0.1 s)$, the total energy decreases smoothly. The kinetic energy is transformed into potential energy, as the front face of the wave steepens and the crest height increases. Then the jet is formed and ejected from the crest $(t \simeq 0.09 s)$. It starts to free-fall down: the potential energy decreases and turns into kinetic energy, which increases subsequently until the jet touches down the forward face of the wave at the time $t \simeq 0.16 \mathrm{~s}$ (Fig. 19a). The potential energy appears to decrease dramatically, due to the low ordinate of the plunging point, the forward face of the wave being almost horizontal. The jet rebounds and the kinetic energy keeps increasing until it reaches a maximum value of $E_{c} \simeq 1.25$ at $t \simeq 0.19 \mathrm{~s}$, corresponding to the beginning of the generation of the splash-up (Fig. 20b and c). More than $60 \%$ of the potential energy is transformed into kinetic energy or dissipated at $t \simeq 0.19 \mathrm{~s}$. Then, the kinetic energy is transferred into potential energy, leading to a fast diminution in magnitude, as the generated splash-up rises higher than the original waveheight (Fig. 20d). The main splash-up starts to be generated: the total energy decreases rapidly, as some energy has to be spent to push the large amount of water. This rapidly diminishing kinetic 
energy diminishing indicates the strength of the wave, as the impact looks spectacular when such a high splash-up is observed. This can be observed through the dotted line plotted in Fig. 23, which shows the time evolution of the potential energy: it stops decreasing and suddenly rises to an extreme value indicating that the main splash-up has reached its highest level. Each decrease in the potential energy is associated with a relative increase in the kinetic energy and vice versa, due to the generation of the successive splashups. The dotted line plotted in Fig. 23 translates this swap process between the kinetic and potential energies. Fig. 23 also indicates that the successive splash-ups rise up high, as the dotted line showing the time evolution of the potential energy passes over the dashed line presenting the time evolution of the kinetic energy. This is due to the very high level reached by the successive splash-ups.

At $t \simeq 0.3 \mathrm{~s}$, when the successive splash-ups fall down and the entrapped gas pockets rise rapidly to the surface (Fig. 20e), the kinetic energy decreases more slowly, as the whole water domain is now rough. The kinetic and potential energies both keep decreasing as the large gas pockets are entrained in the water. About $40 \%$ of the total pre-breaking wave energy is dissipated at $t \simeq 0.3 \mathrm{~s}$. Almost $40 \%$ of the potential energy and $55 \%$ of the kinetic energy remain in the wave. Then, the energy is dissipated slowly. At $t \simeq 1.5 \mathrm{~s}$, which is almost five wave periods of time, about $35 \%$ of the total pre-breaking wave 
energy, $40 \%$ of the kinetic energy and $30 \%$ of the potential energy remain in the numerical domain.

The total wave energy never stops decreasing, unlike the potential and kinetic energies. It first slowly decreases due to the viscosity. It also decreases because, at the beginning of the simulation, the air is set at rest, so when the wave starts propagating, some energy is dissipated to put the air into motion. About $13 \%$ of the total pre-breaking energy is observed to be dissipated before the jet impact. This is in accordance with the $10 \%$ found from the computations of Chen et al. (1999). This tends to prove that the flow is no more irrotational when the wave starts to steepen. Then, it can be clearly seen that it is mainly dissipated during the wave breaking process: about $40 \%$ of the total prebreaking wave energy is dissipated at $t \simeq 0.3 \mathrm{~s}$. Almost $50 \%$ of the potential energy and $65 \%$ of the kinetic energy remain in the wave at this time. Then, the energy is dissipated slowly. At $t \simeq 1 \mathrm{~s}$, which is about three wave periods of time, about $40 \%$ of the total pre-breaking wave energy, $45 \%$ of the kinetic energy and $30 \%$ of the potential energy remain in the numerical domain. These values should to be compared with the work of Chen et al. (1999), who found that around $30 \%$ of the kinetic energy and $5 \%$ of the potential energy remain in the wave, more than $80 \%$ of the total pre-breaking wave energy being dissipated at this time. The kinetic energy is shown to reach a maximum value of $E_{c} \simeq 1.05$, which is lower than what we found. However, it 
has to be kept in mind that the simulation presented by Chen et al. (1999) was run with greater viscosity and density ratios $\left(\mu_{a} / \mu_{w}=0.4\right.$ and $\left.\rho_{a} / \rho_{w}=10^{-2}\right)$ than those we considered $\left(\mu_{a} / \mu_{w}=1.85 \times 10^{-2}\right.$ and $\left.\rho_{a} / \rho_{w}=1.1768 \times 10^{-3}\right)$, considering the breaking of a wave in deep water at a lower Reynolds number $\left(R e=10^{4}\right)$. The flow appeared then to be damped more quickly, as can be observed from the figures shown by Chen et al. (1999), due to the higher viscosities and densities ratios. Moreover, the deep depth configuration they considered means that the momentum was spread in a greater quantity of water than the shallower configuration we chose.

After $t \simeq 0.5 \mathrm{~s}$, the propagating vortices move out of the numerical domain on the right side to re-enter on the left side. These vortices come and interact with the two rising counter-rotative vortices (Figs. 18). In reality, this should not happen, as the co-rotative vortices propagate towards the shore-line, leaving behind the two counter-rotative vortices. Moreover, we chose to examine shallow water configurations. For the very low viscosities and densities of air and water: these parameters allow velocity fluctuations to last for a long time, constrained between the bottom of the numerical domain and the free surface (Sous et al., 2004). It is different from the case of a wave breaking over a sloping beach, forced to dissipate all its energy. For these reasons the water keeps waving in the numerical domain with a strongly distorted interface. This is why there is a clear change of dissipation regime, as there seems to be an in- 
flexion point in the curve plotting the total wave energy (Fig. 23) at this time. More than $70 \%$ of the total pre-breaking wave energy is finally observed to be dissipated after seven wave periods of time. Some similar descriptions can be given for the two other cases we chose to detail, with the same swapping between the kinetic and potential energies. It can also be highlighted that, in the three cases detailed previously, the maximum of the total pre-breaking wave energy is dissipated during the splash-ups, an average value of $40 \%$ of the total pre-breaking wave energy being estimated from the numerical results. This observation is in a very good agreement with the results of Lamarre and Melville (1991) who showed experimentally that up to $50 \%$ of the wave energy is spent in entraining air and resisting buoyancy forces, with the turbulent behavior of the entrained and rising bubbles increasing this dissipation process. As shown by Chen et al. (1999), it is observed that the curve of the time evolution of the total energy gives three distinct slopes indicating different regimes of wave energy decay. During the wave propagation, steepening and breaking processes, the total wave energy follows an exponential decay, with two different decay rates before the jet ejection and before the jet impact. The main observation we make is that the dependence of the decay of the total energy is found to be different from the observation of Chen et al. (1999). They highlighted a $t^{-1}$ dependence, whereas we find a general $t^{-0.3}$ dependence from the times of the generation of the splash-ups. 
As suggested, three-dimensional simulations exhibit different features from two-dimensional simulations. This has been discussed in terms of the behavior of vortices. We illustrate this point by examining the total energy dissipation. The time evolution of the total energy, normalized by its initial value is shown in Fig. 24. The solid line stands for the three-dimensional result, compared with the two-dimensional result represented by the dashed line.

Figure 24 should be here

It is obvious that two-dimensional turbulence is less dissipative than threedimensional turbulence. So, as expected, a very clear difference can be observed in terms of total wave energy decay, the main features being comparable but of different magnitudes. It can be seen than the discrepancy starts at the time of the impact of the jet on the forward face of the wave $(t \simeq 0.17 \mathrm{~s})$ and increases with the turbulence generation, resulting from the splash-ups and the vortices. At $t \simeq 0.3 \mathrm{~s}$, when the successive splash-ups entrain the gas pockets, about $50 \%$ of the total pre-breaking wave energy is dissipated in the two-dimensional simulation, compared with the $45 \%$ dissipated in the three-dimensional simulation. The decay rate is much greater for the threedimensional result as the turbulence generation process is mainly observed at this time of the breaking phenomenon. The pockets of gas are entrained in the water and dislocated before rising to the free surface. At the time when all these large structures have disappeared, a large amount of water has been 
put into three-dimensional turbulent motion.

Two and a half wave periods later $(t \simeq 0.9 s)$, almost $30 \%$ of the total pre-breaking wave energy remains in the two-dimensional numerical domain, whereas almost $20 \%$ of the total pre-breaking wave energy remains in the three-dimensional numerical domain. However, both curves seem to exhibit the same decay rate at the end of the breaking process. The $10 \%$ difference between the two simulations tends to prove that the flow remains mainly twodimensional. This would need to be checked more precisely and has to be verified with a longer time course of simulation.

\section{Conclusion}

In this paper, the physical processes associated with three-dimensional plunging breaking waves are described and investigated using an original numerical model based on a Large Eddy Simulation modelling of turbulence.

The present numerical model gives very satisfactory results in the validation section for a propagation problem and a dam-break configuration. We proved the ability of the numerical tool to conserve both the mass and energy along the simulations. We also successfully compared the VOF-PLIC and TVD methods in the description of the free surface. We have shown a very good agreement in both profiles and characteristic times for the simulation of the 
breaking of a dam on a wet bottom, compared with the experimental data.

The numerical tool accurately reproduced the overturning motion, the splashup occurrence and the complicated dynamics generated under plunging breaking waves. A detailed description is given in terms of free surface and velocity field. Some complex features have been illustrated. The numerical results highlight the major role of the air entrainment phenomenon in the energy dissipation process. Due to its role in the turbulence generation, the air entrainment has therefore to be taken into account during numerical simulations of breaking waves. A substantial improvement of the usual numerical methods is presented, as they have been able to take into account usually neglected processes such as entrained spinning gas pockets. We have shown that the numerical tool is relevant for the use of numerical experiments to study physical processes beyond the reach of experimental methods. The model is a reliable tool for describing complicated two-phase flow features, such as vortices and air entrainment. This kind of modelling will be of great interest for engineering design and coastal studies.

We proved the capacity of the numerical tool to deal with a really complicated two-phase flow problem applied to coastal application. Additional work and investigations are being undertaken to improve the numerical methods and modelling of turbulence to gain precision for the flow description. Some more systematical work needs to be carried out to study the transition from one 
type of breaker to another and to classify the occurrence of induced vortices or describe some of the geometrical characteristics of the plunging jet. In particular, some work is dedicated to evaluate the rate of air entrained as a function of time, depending on the breaker configuration. The numerical model is also employed to simulate regular waves shoaling and breaking over sloping beaches and coastal structures. Transport equations are already included in the numerical tool to work on the impact of breaking waves on the sediment transport processes. Taking into account the mixture of air and water is one of the challenges of the coming years.

\section{Acknowledgements}

The authors would like to acknowledge the financial and scientific support of the french INSU - CNRS (Institut National des Sciences de l'Univers -

Centre National de la Recherche Scientifique) program "Programme Atmosphère-Océan Multi-échelle" (PATOM) and the IDRIS-CNRS (Institut du Développement et des Ressources en Informatique Scientifique) and CINES (Centre Informatique National de l'Enseignement Supérieur) for their material support (project ter 2237). We also thank Pr. Mohamed Rady, for his critical reading that guided us in improving this article. 


\section{References}

S. Abadie. Numerical modelling of the flow generated by plunging breakers. In Proceedings Coastal Dynamics 2001, pages 202-211, 2001.

S. Abadie, J.-P. Caltagirone, and P. Watremez. Mécanisme de génération du jet secondaire ascendant dans un déferlement plongeant. C. R. Mécanique, 326:553-559, 1998.

P. Angot, C. Bruneau, and P. Fabrie. A penalization method to take into account obstacles in incompressible viscous flows. Nümerische Mathematik, 81(4):497-520, 1999.

J. A. Battjes. Surf-zone dynamics. Annu. Rev. Fluid Mech., 20:257-293, 1988.

B. Biausser, S. T. Grilli, P. Fraunie, and R. Marcer. Numerical analysis of the internal kinematics and dynamics of three-dimensional breaking waves on slopes. Intl. J. Offshore and Polar Engng., 14(4):247-256, 2004.

A. Blanco-Alvarez. Quelques aspects de l'écoulement d'un fluide visqueux autour d'une bulle déformable : une analyse par simulation directe. $\mathrm{PhD}$ thesis, Institut National Polytechnique de Toulouse, 1995.

P. Bonmarin. Geometric properties of deep-water breaking waves. J. Fluid Mech, 209:405-433, 1989.

J. U. Brackbill, B. D. Kothe, and C. Zemach. A continuum method for modelling surface tension. J. Comput. Phys., 100:335-354, 1992.

S. F. Bradford. Numerical simulation of surf zone dynamics. J. of Waterway, 
Port, Coastal, and Ocean Eng., 126(1):1-13, 2000.

H. Chanson, S. Aoki, and M. Maruyama. Unsteady air bubble entrainment and detrainment at a plunging breaker: dominant time scales and similarity of water level variations. Coastal Eng., 46:139-157, 2002.

H. Chanson and J.-F. Lee. Plunging jet characteristics of plunging breakers. Coastal Eng., 31:125-141, 1997.

G. Chen, C. Kharif, S. Zaleski, and J. J. Li. Two-dimensional navier-stokes simulation of breaking waves. Phys. Fluids, 11:121-133, 1999.

E. D. Christensen and R. Deigaard. Large eddy simulation of breaking waves. Coastal Eng., 42:53-86, 2001.

E. D. Christensen, D.-J. Walstra, and N. Emerat. Vertical variation of the flow across the surf zone. Coastal Eng., 45:169-198, 2002.

E. D. Cokelet. Breaking waves. Nature, 267:769-774, 1977.

E. D. Cokelet. Mechanics of Wave-Induced Forces on Cylinders, chapter Breaking waves, the plunging jet and interior flow-field, pages 287-301. Ed. T. L. Shaw, 1979.

E. David. Modélisation des écoulements compressibles et hypersoniques. $\mathrm{PhD}$ thesis, Institut National Polytechnique de Grenoble, 1993.

J. H. Duncan, H. Qiao, V. Philomin, and A. Wenz. Gentle spilling breakers: crest profile evolution. J. Fluid Mech, 39:191-222, 1999.

M. Fortin and R. Glowinski. Méthodes de lagrangien augmenté. Application à 
la résolution numérique de problèmes aux limites. Dunod, Paris, 1982.

C. J. Galvin. Breaker type classification on three laboratory beaches. J. of Geophysical Research, 73:3651-3659, 1968.

S. T. Grilli, R. Gilbert, P. Lubin, S. Vincent, D. Astruc, D. Legendre, M. Duval, D. Drevard, P. Fraunié, and S. Abadie. Numerical modeling and experiments for solitary wave shoaling and breaking over a sloping beach. In Proc. $14^{\text {th }}$ Int. Offshore and Polar Engng. Conf., volume 3, pages 306-312, 2004 .

D. Gueyffier, A. Nadim J. Li, S. Scardovelli, and S. Zaleski. Volume of fluid interface tracking with smoothed surface stress methods for three-dimensional flows. J. of Comp. Phys., 152:423, 1999.

S. Guignard, R. Marcer, V. Rey, C. Kharif, and P. Fraunié. Solitary wave breaking on sloping beaches : 2-d two phase flow numerical simulation by sl-vof method. Eur. J. Mech. B - Fluids, 20:57-74, 2001.

I. Gustafsson. On first and second order symmetric factorization methods for the solution of elliptic difference equations. Chalmers University of Technology, 1978.

P. Helluy, F. Gollay, S. T. Grilli, N. Seguin, P. Lubin, J.-P. Caltagirone, S. Vincent, D. Drevard, and R. Marcer. Numerical simulations of wave breaking. Mathematical Modelling and Numerical Analysis, 39(3):591-608, 2005.

P. D. Hieu, T. Katsutoshi, and V. T. Ca. Numerical simulation of breaking 
waves using a two-phase flow model. Applied Mathematical Modelling, 28 (11):983-1005, 2004.

C. W. Hirt and B. D. Nichols. Volume of fluid (vof) methods for the dynamics of free boundaries. J. Comput. Phys., 39:201-255, 1981.

J. G. Hnat and J. D. Buckmaster. Spherical cap bubbles and skirt formation. Phys. Fluids, 19:182-194, 1976.

A. Iafrati and E. F. Campana. A domain decomposition approach to compute wave breaking (wave breaking flows). Int. J. Numer. Meth. Fluids, 41:419$445,2003$.

A. Iafrati, A. Di Mascio, and E. F. Campana. A level set technique applied to unsteady free surface flows. Int. J. Numer. Meth. Fluids, 35:281-297, 2001.

P. C. M. Jansen. Laboratory observations of the kinematics in the aerated region of breaking waves. Coastal Eng., 9:453-477, 1986.

K. Khadra, P. Angot, S. Parneix, and J.-P. Caltagirone. Fictitious domain approach for numerical modelling of navier-stokes equations. Int. J. Numer. Meth. Fluids, 34:651-684, 2000.

E. Labourasse, D. Lacanette, A. Toutant, P. Lubin, S. Vincent, O. Lebaigue, J.-P. Caltagirone, and P. Sagaut. Towards large eddy simulation of isothermal two-phase flows: governing equations and a priori tests. Under correction for Int. J. Multiphase Flow, 2004.

B. Lafaurie, C. Nardone, R. Scardovelli, S. Zaleski, and G. Zanetti. Modelling 
merging and fragmentation in multiphase flows with surfer. J. Comput. Phys., 113:134, 1994.

E. Lamarre and W. K. Melville. Air entrainment and dissipation in breaking waves. Nature, 351:469, 1991.

O. Lebaigue, C. Duquennoy, and S. Vincent. Rise of a spherical cap bubble in a stagnant liquid. Multiphase Science and Technology, 16(1-3):1-4, 2004.

J.-J. Lee, J. E. Skjelbreia, and F. Raichlen. Measurements of velocities in solitary waves. J. of Waterway, Port, Coastal, and Ocean Eng., WW2(108): 200-218, 1982.

C. M. Lemos. Wave breaking: A Numerical Study, volume 71 of Lecture Notes in Engineering. Springer Verlag, 1992.

R. J. LeVeque. Numerical methods for conservation laws. Lectures in Mathematics. Birkhauser, Zurich, 1992.

C. Lin and H. H. Hwung. External and internal flow fields of plunging breakers. Experiments in Fluids, 12:229-237, 1992.

P. Lin and P. L.-F. Liu. A numerical study of breaking waves in the surf zone. J. Fluid Mech, 359:239-264, 1998a.

P. Lin and P. L.-F. Liu. Turbulence transport, vorticity dynamics, and solute mixing under plunging breaking waves in surf zone. J. of Geophysical Research, 103(C8):15,677-15,694, 1998b.

P. L.-F. Liu. Free surface tracking methods and their applications to wave 
hydrodynamics, volume 5 of Advances in Coastal and Ocean Eng., pages 213-240. World Scientific, 1999.

P. Lubin. Large Eddy Simulation of plunging breaking waves. $\mathrm{PhD}$ thesis, Université Bordeaux I, 2004. in English.

P. Lubin and H. Lemonnier. Propagation of solitary waves in constant depths over horizontal beds. Multiphase Science and Technology, 16(1-3):237-248, 2004 .

P. Lubin, S. Vincent, J.-P. Caltagirone, and S. Abadie. Fully three-dimensional direct simulation of a plunging breaker. C. R. Mécanique, 331(7):495-501, 2003.

P. Lubin, S. Vincent, J.-P. Caltagirone, and S. Abadie. Three-dimensional large eddy simulation of vortices induced by plunging breaking waves. In World Scientific, editor, ASCE 29th International Conference on Coastal Engineering, pages 253-262, 2004.

R. L. Miller. Role of vortices in surf zone predictions : sedimentation and wave forces, chapter 24, pages 92-114. Soc. Econ. Paleontol. Mineral. Spec. Publ., R. A. Davis and R. L. Ethington, 1976.

H. Mutsuda and T. Yasuda. Numerical simulation of turbulent air-water mixing layer within surf-zone. In Proc. $27^{\text {th }}$ Int. Conf. Coastal Eng., pages $755-768,2000$.

K. Nadaoka, M. Hino, and Y. Koyano. Structure of the turbulent flow field 
under breaking waves in the surf zone. J. Fluid Mech, 204:359-387, 1989.

K. Nadaoka and T. Kondoh. Laboratory measurements of velocity field structure in the surf zone by ldv. Coastal Eng. J. in Japan, 25:125-145, 1982.

A. L. New, P. McIver, and D. H. Peregrine. Computations of overturnings waves. J. Fluid Mech, 150:233-251, 1985.

S. Osher and J. A. Sethian. Fronts propagating with curvature dependent speed: algorithm based on hamilton-jacobi formulations. J. Comp. Phys., 79:12-49, 1988.

S. V. Patankar. Numerical heat transfer and fluid flow. Hemisphere Publishing Corporation, New York, 1990.

D. H. Peregrine. The fascination of fluid mechanics. J. Fluid Mech, 106:59-80, 1981.

D. H. Peregrine. Breaking waves on beaches. Annu. Rev. Fluid Mech., 15: 149-178, 1983.

P. Sagaut. Large Eddy Simulation for incompressible flows - An introduction. Springer Verlag, 1998.

P. Sagaut and R. Grohens. Discrete filters for large eddy simulation. Int. J. Numer. Meth. Fluids, 31:1195-1220, 1999.

T. Sakai, T. Mizutani, H. Tanaka, and Y. Tada. Vortex formation in plunging breaker. In Proc. ICCE, pages 711-723, 1986.

R. Scardovelli and S. Zaleski. Direct numerical simulation of free-surface and 
interfacial flow. Annu. Rev. Fluid Mech., 31:567-603, 1999.

C. Song and A. I. Sirviente. A numerical study of breaking waves. Phys. Fluids, 16(7):2649-2667, 2004.

D. Sous, N. Bonneton, and J. Sommeria. Turbulent vortex dipoles in a shallow water layer. Phys. Fluids, 16(8):2886-2898, 2004.

P. K. Stansby, A. Chegini, and T. C. D. Barnes. The initial stages of dambreak flows. J. Fluid Mech, 374:407-424, 1998.

I. A. Svendsen and U. Putrevu. Surf-zone hydrodynamics, volume 2 of Advances in Coastal and Ocean Eng., pages 1-78. World Scientific, 1996.

K. Takikawa, F. Yamada, and K. Matsumoto. Internal characteristics and numerical analysis of plunging breaker on a slope. Coastal Eng., 31(1-4): 143-161, 1997.

S. O. Unverdi and G. T. Tryggvason. A front-tracking method for viscous, incompressible, multi-fluid flows. J. Comp. Phys., 100:25-37, 1992.

H. Uzawa. Iterative method for concave programming. K. J. Arrow, L. Hurwicz and H. Uzawa, editors, Studies in linear and Nonlinear Programming, Stanford University Press, Stanford, CA, 1958.

H. A. VanDerVorst. Bi-cgstab: a fast and smoothly converging variant of bicg for the solution of non-symmetric linear systems. SIAM J. Sci. Stat. Comput., 13:631-644, 1992.

S. Vincent and J.-P. Caltagirone. Efficient solving method for unsteady in- 
compressible interfacial flow problems. Int. J. Numer. Meth. Fluids, 30: 795-811, 1999.

S. Vincent and J.-P. Caltagirone. A one cell local multigrid method for solving unsteady incompressible multi-phase flows. J. Comput. Phys., 163:172-215, 2000.

S. Vincent and J.-P. Caltagirone. Parasitic currents induced by surface tension. Multiphase Science and Technology, 16(1-3):67-72, 2004.

S. Vincent, J.-P. Caltagirone, and O. Lebaigue. Poiseuille two-phase flow. Multiphase Science and Technology, 16(1-3):97-100, 2004a.

S. Vincent, J.-P. Caltagirone, P. Lubin, and T. N. Randrianarivelo. An adaptative augmented lagrangian method for three-dimensional multi-material flows. Computers and Fluids, 33:1273-1289, 2004b.

T. Vinje and P. Brevig. Numerical simulation of breaking waves. J. Adv. Water Resour., 4:77-82, 1981.

Y. Watanabe and H. Saeki. Three-dimensional large eddy simulation of breaking waves. Coastal Eng. J. in Japan, 41(2):281-301, 1999.

Y. Watanabe and H. Saeki. Velocity field after wave breaking. Int. J. Numer. Meth. Fluids, 39:607-637, 2002.

T. Yasuda, H. Mutsuda, and N. Mizutani. Kinematics of overturning solitary waves and their relations to breaker types. Coastal Eng., 29:317-346, 1997.

T. Yasuda, H. Mutsuda, N. Mizutani, and H. Matsuda. Relationships of plung- 
ing jet size to kinematics of breaking waves with spray and entrained air bubbles. Coastal Eng. J. in Japan, 41(2):269-280, 1999.

D. Zhang and T. Sunamura. Conditions for the occurence of vortices induced by breaking waves. Coastal Eng. J. in Japan, 33(2):145-155, 1990.

Q. Zhao, S. Armfield, and K. Tanimoto. Numerical simulation of breaking waves by multi-scale turbulence model. Coastal Eng., 51:53-80, 2004.

Q. Zhao and K. Tanimoto. Numerical simulation of breaking waves by large eddy simulation and vof method. In Proc. of the 26th Int. Conf. Coastal Eng., volume 1, pages 892-905. ASCE, 1998. 
List of tables 


\begin{tabular}{|c|c|c|c|c|}
\hline Schemes & \multicolumn{2}{|c|}{ VOF-PLIC } & \multicolumn{2}{c|}{ TVD } \\
\hline Surface tension & With $\sigma$ & Without $\sigma$ & With $\sigma$ & Without $\sigma$ \\
\hline$z_{\text {fin }}(m)$ & 0.33054 & 0.33054 & 0.3339 & 0.3339 \\
\hline Error $(\mathrm{m})$ & $-4.68 \times 10^{-3}$ & $-4.68 \times 10^{-3}$ & $-1.32 \times 10^{-3}$ & $-1.32 \times 10^{-3}$ \\
\hline Error $(\%)$ & $-1.4 \%$ & $-1.4 \%$ & $-4 \times 10^{-1} \%$ & $-4 \times 10^{-1} \%$ \\
\hline$x_{\text {fin }}(m)$ & 80.85 & 80.89 & 81.13 & 81.17 \\
\hline Error $(\mathrm{m})$ & 0.753 & 0.713 & 0.473 & 0.433 \\
\hline Error $(\%)$ & $0.92 \%$ & $0.87 \%$ & $0.58 \%$ & $0.53 \%$ \\
\hline
\end{tabular}

Table 1

Numerical errors calculated for the solitary wave coordinates.

\begin{tabular}{|c|c|c|c|c|}
\hline Schemes & \multicolumn{2}{|c|}{ VOF-PLIC } & \multicolumn{2}{c|}{ TVD } \\
\hline Surface tension & With $\sigma$ & Without $\sigma$ & With $\sigma$ & Without $\sigma$ \\
\hline volume $_{\text {fin }}\left(\mathrm{m}^{2}\right)$ & 3.109 & 3.110 & 3.114 & 3.114 \\
\hline Error $\left(\mathrm{m}^{2}\right)$ & $-6.9 \times 10^{-3}$ & $-6 \times 10^{-3}$ & $-2 \times 10^{-3}$ & $-2 \times 10^{-3}$ \\
\hline Error $(\%)$ & $-2.2 \times 10^{-1} \%$ & $-2 \times 10^{-1} \%$ & $-6.42 \times 10^{-2} \%$ & $-6.42 \times 10^{-2} \%$ \\
\hline
\end{tabular}

Table 2

Numerical errors calculated for the solitary wave mass conservation. 
Table 3

\begin{tabular}{|l|l|l|l|l|}
\hline & \multicolumn{4}{|c|}{$d / L$} \\
\hline$H / L$ & 0.1 & 0.13 & 0.17 & 0.2 \\
\hline 0.06 & $\mathrm{WP}$ & $\mathrm{NB}$ & $\mathrm{NB}$ & $\mathrm{NB}$ \\
\hline 0.07 & $\mathrm{WP}$ & $\mathrm{WP}$ & $\mathrm{NB}$ & $\mathrm{NB}$ \\
\hline 0.08 & $\mathrm{PL}$ & $\mathrm{WP}$ & $\mathrm{WP}$ & $\mathrm{NB}$ \\
\hline 0.09 & $\mathrm{PL}$ & $\mathrm{PL}$ & $\mathrm{WP}$ & $\mathrm{WP}$ \\
\hline 0.1 & $\mathrm{PL}$ & $\mathrm{PL}$ & $\mathrm{PL}$ & $\mathrm{WP}$ \\
\hline 0.11 & $\mathrm{PL}$ & $\mathrm{PL}$ & $\mathrm{PL}$ & $\mathrm{PL}$ \\
\hline 0.12 & $\mathrm{PL}$ & $\mathrm{PL}$ & $\mathrm{PL}$ & $\mathrm{PL}$ \\
\hline 0.13 & $\mathrm{CO}$ & $\mathrm{PL}$ & $\mathrm{PL}$ & $\mathrm{PL}$ \\
\hline 0.14 & $\mathrm{CO}$ & $\mathrm{CO}$ & $\mathrm{PL}$ & $\mathrm{PL}$ \\
\hline 0.15 & $\mathrm{CO}$ & $\mathrm{CO}$ & $\mathrm{CO}$ & $\mathrm{PL}$ \\
\hline 0.16 & $\mathrm{CO}$ & $\mathrm{CO}$ & $\mathrm{CO}$ & $\mathrm{CO}$ \\
\hline 0.17 & $\mathrm{CO}$ & $\mathrm{CO}$ & $\mathrm{CO}$ & $\mathrm{CO}$ \\
\hline 0.18 & $\mathrm{CO}$ & $\mathrm{CO}$ & $\mathrm{CO}$ & $\mathrm{CO}$ \\
\hline
\end{tabular}

Values of the initial steepnesses, $H / L$, and dispersion parameters, $d / L$, for a constant wavelength. Two-dimensional simulations. NB: non-breaking wave; WP: weak plunging breaker; PL: plunging breaker; CO: collapsing breaker.

\begin{tabular}{|c|c|c|c|}
\hline Test-case & $\frac{H}{L}=0.10, \frac{d}{L}=0.10$ & $\frac{H}{L}=0.13, \frac{d}{L}=0.13$ & $\frac{H}{L}=0.13, \frac{d}{L}=0.17$ \\
\hline Celerity $\left(m . s^{-1}\right)$ & 0.295 & 0.324 & 0.351 \\
Wave period $(s)$ & 0.339 & 0.308 & 0.285 \\
Reynolds number & 29500 & 32400 & 35000 \\
\hline
\end{tabular}

Table 4

Characteristics of the presented plunging breaking cases. 


\section{Table 5}

\begin{tabular}{|l|l|l|l|l|}
\hline & \multicolumn{4}{|c|}{$d / L$} \\
\hline$H / L$ & 0.1 & 0.13 & 0.17 & 0.2 \\
\hline 0.06 & $*$ & $\mathrm{NB}$ & $\mathrm{NB}$ & $\mathrm{NB}$ \\
\hline 0.07 & $*$ & $*$ & $\mathrm{NB}$ & $\mathrm{NB}$ \\
\hline 0.08 & $*$ & $*$ & $*$ & $\mathrm{NB}$ \\
\hline 0.09 & $\bullet$ & $*$ & $*$ & $*$ \\
\hline 0.1 & $\bullet$ & $\bullet$ & $*$ & $*$ \\
\hline 0.11 & $\bullet$ & $\bullet$ & $\bullet$ & $\bullet$ \\
\hline 0.12 & $\bullet$ & $\bullet$ & $\bullet$ & $\bullet$ \\
\hline 0.13 & $\bullet$ & $\bullet$ & $\bullet$ & $\bullet$ \\
\hline 0.14 & $\circ$ & $\bullet$ & $\bullet$ & $\bullet$ \\
\hline 0.15 & $\bullet$ & $\circ$ & $\bullet$ & $\bullet$ \\
\hline 0.16 & $\circ$ & $\circ$ & $\bullet$ & $\bullet$ \\
\hline 0.17 & $\circ$ & $\bullet$ & $\bullet$ & $\bullet$ \\
\hline 0.18 & $\circ$ & $\bullet$ & $\bullet$ & $\bullet$ \\
\hline
\end{tabular}

Types of vortices observed depending on the values of the initial steepnesses, $H / L$, and dispersion parameters, $d / L$, for a constant wavelength. Two-dimensional simulations. NB: non-breaking wave; $*$ : only co-rotative vortices; $\circ$ : only counter-rotative vortices; $\bullet$ : both co-rotative and counter-rotative vortices. 
List of figures 


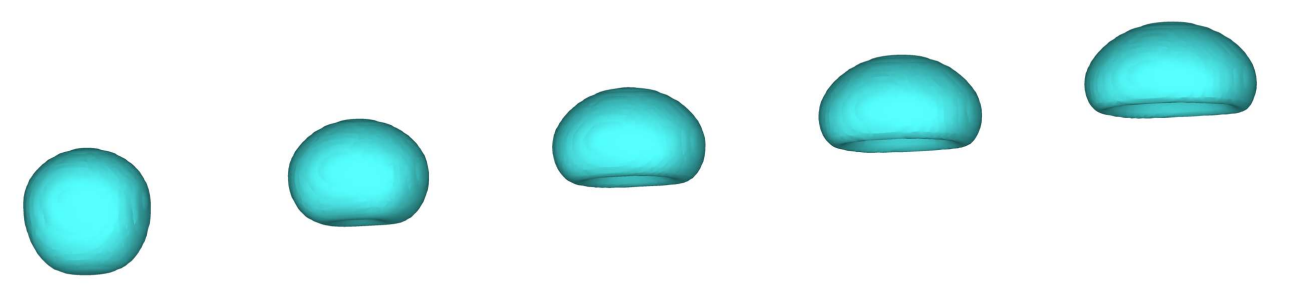

Fig. 1. Rising bubble at $t=0.02 s, 0.04 s, 0.06 s, 0.08 s, 0.1 s$, from left to right.

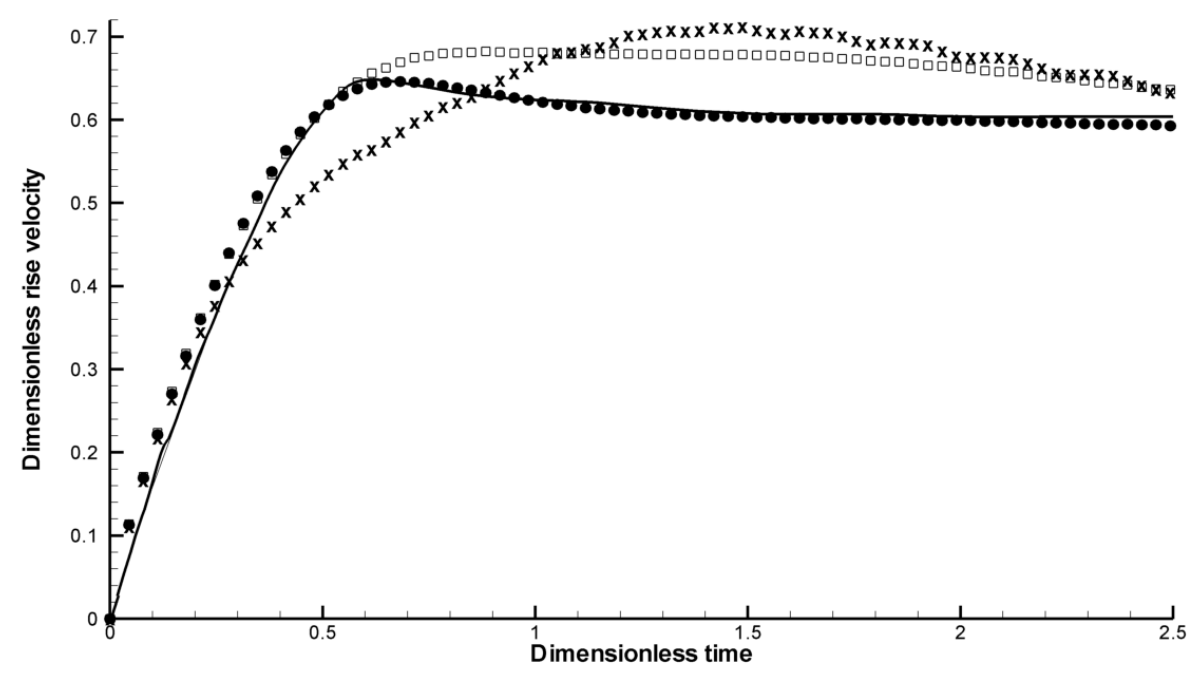

Fig. 2. Comparison between Direct Numerical Simulation (solid line) from Blanco-Alvarez (1995) and our numerical results: • fine grid $69 \times 69 \times 115$, $\square$ intermediate grid $35 \times 35 \times 56, \times$ coarse grid $18 \times 18 \times 99$. 


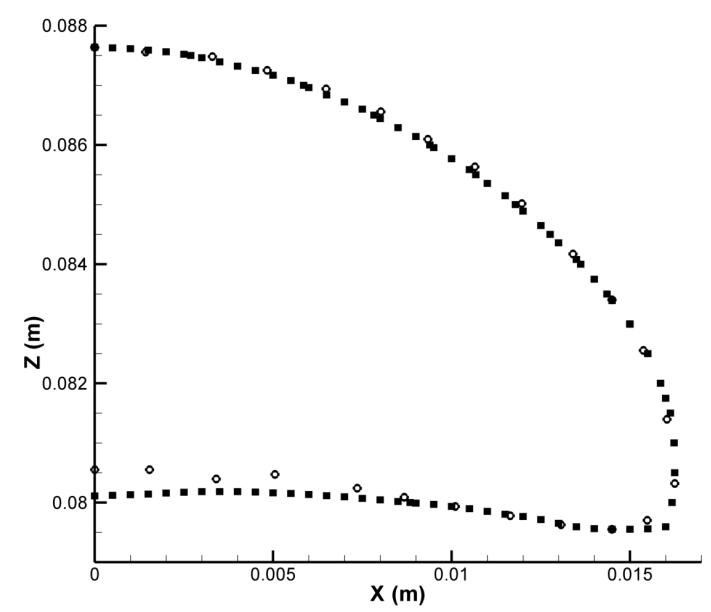

Fig. 3. Comparison between experimental free surface profiles (o) from Hnat and Buckmaster (1976) and three-dimensional numerical results (ם) for the final shape of a rising bubble.

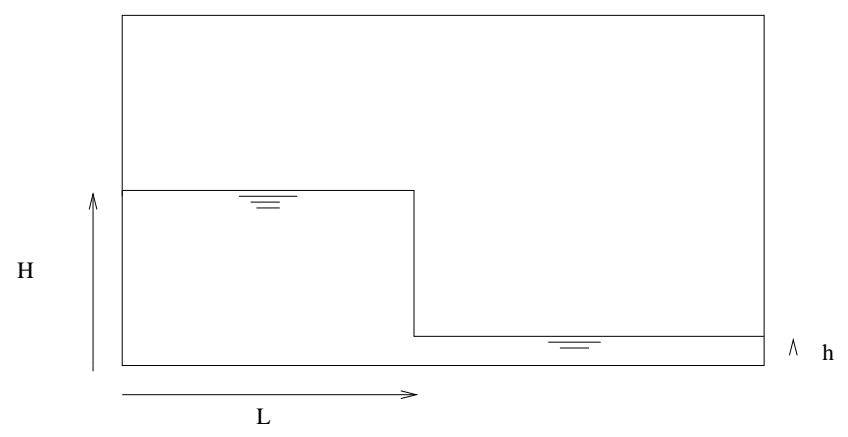

Fig. 4. Sketch of the initial condition for the dam-break problem. 


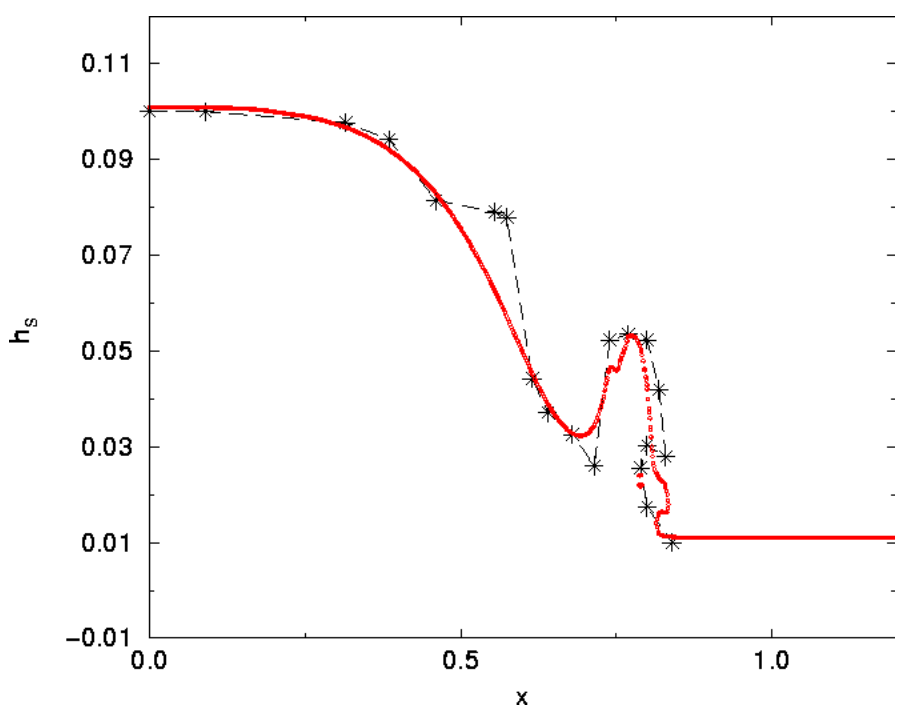

(a) $\mathrm{t}=0.24 \mathrm{~s}$

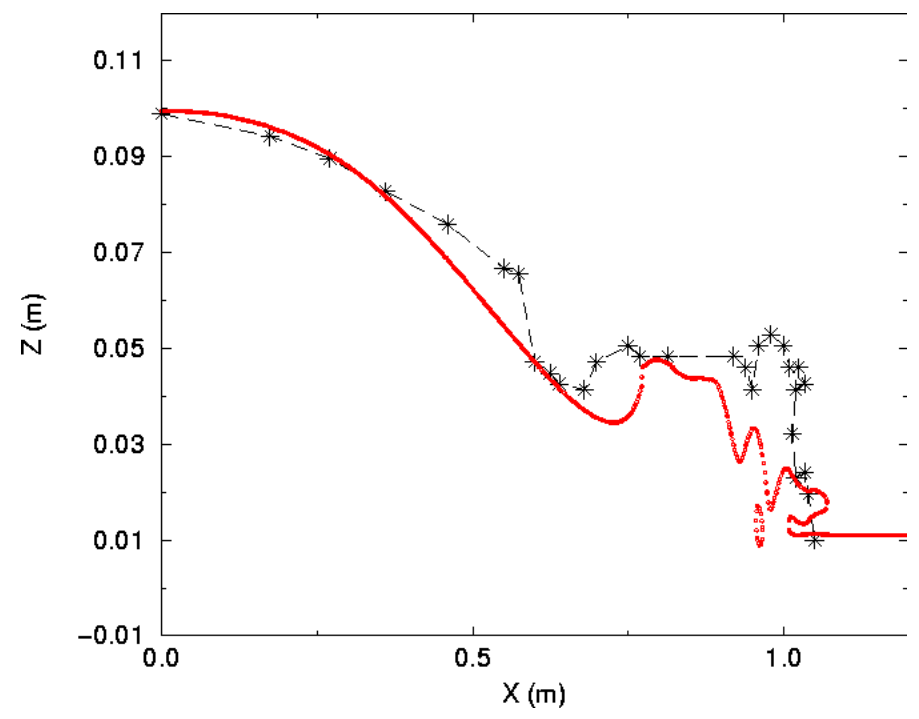

(b) $\mathrm{t}=0.40 \mathrm{~s}$

Fig. 5. Comparison between experimental free surface profiles $(*)$ from Stansby et al. (1998) and three-dimensional numerical results (solid line). 


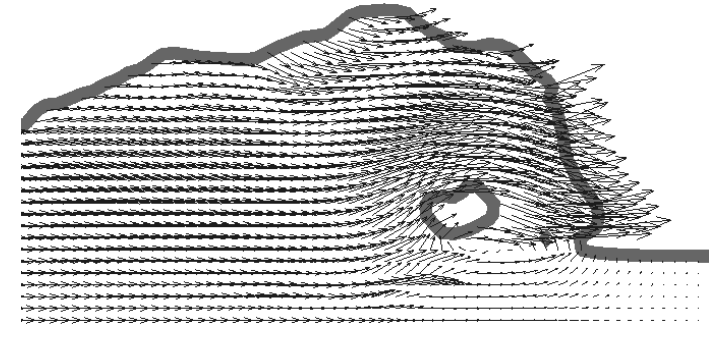

(a) Water

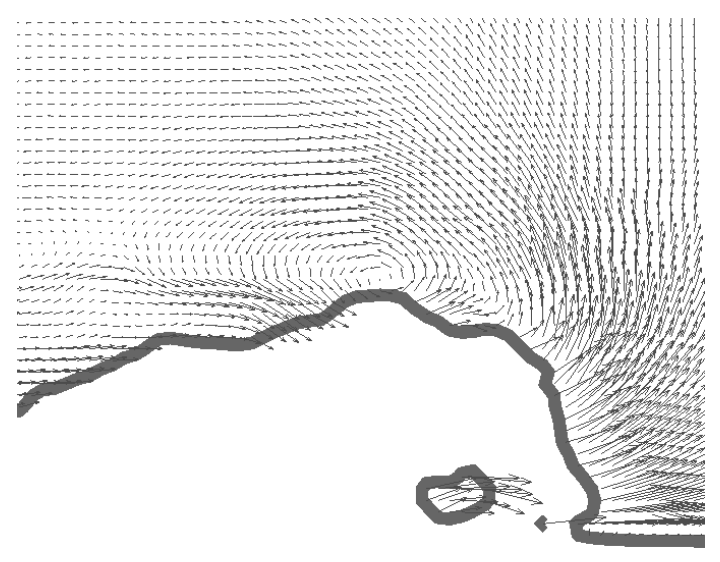

(b) Air

Fig. 6. Velocity field in both media. $t=0.24 s, C=0.5$.

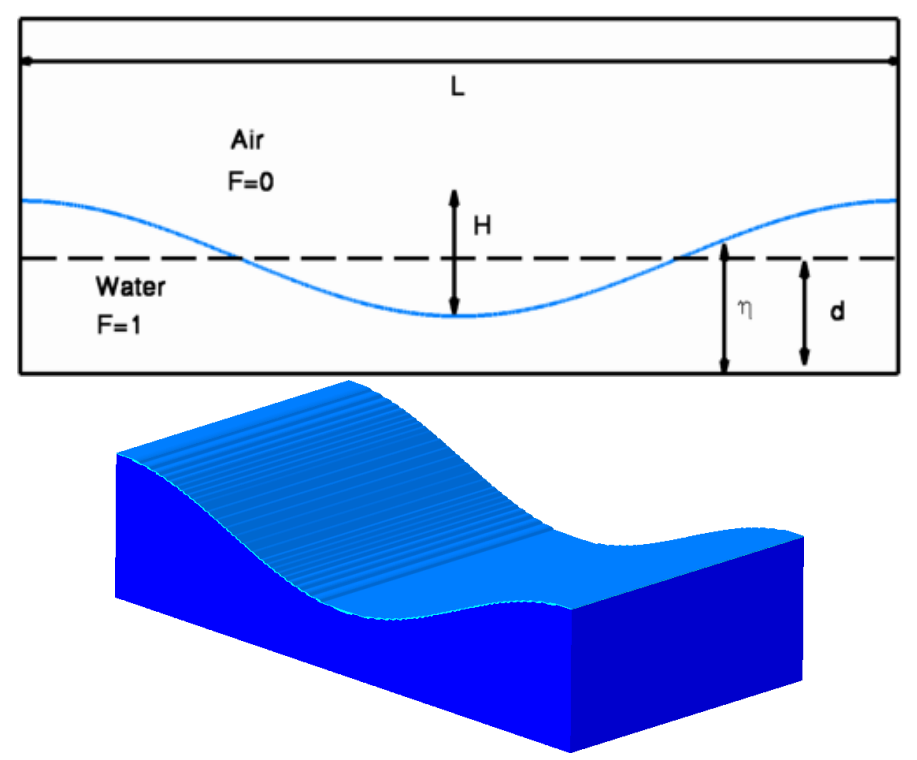

Fig. 7. Sketch of the initial condition for the unstable periodic sinusoidal wave of large amplitude (2D/3D). $\frac{H}{L}=0.13, \frac{d}{L}=0.17, t=0 s, C \geq 0.5$. 

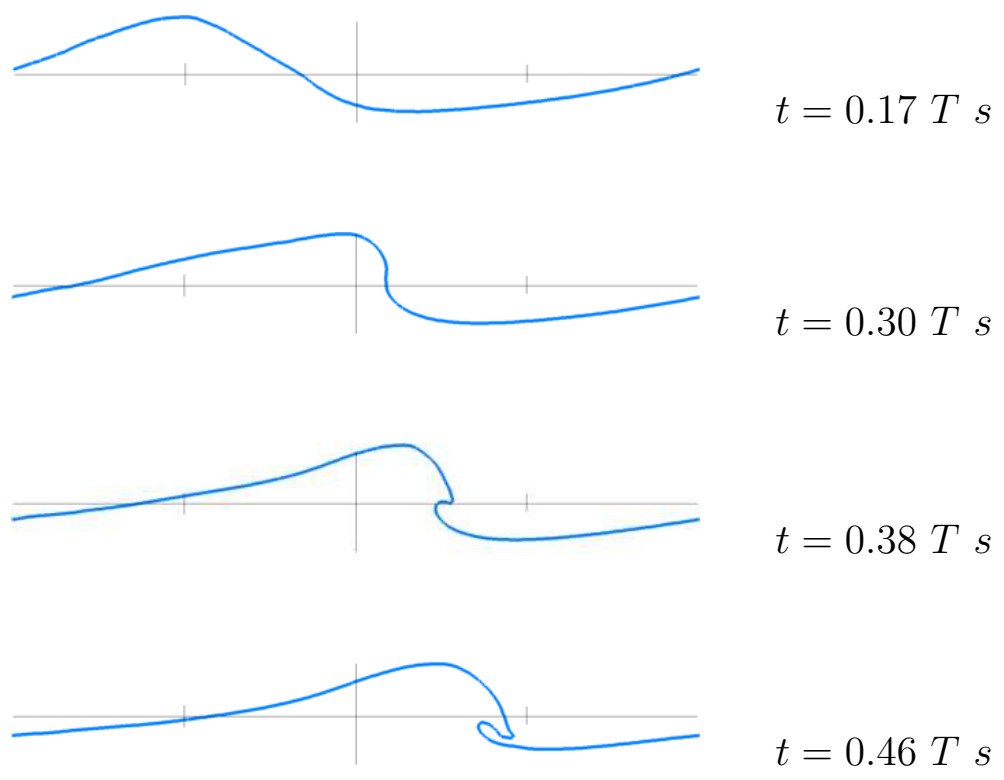

(c) Present study, $C=0.5$

Fig. 8. Time evolution of the overturning motion. Sections taken from a 3D simulation. $\frac{H}{L}=0.13, \frac{d}{L}=0.13$.

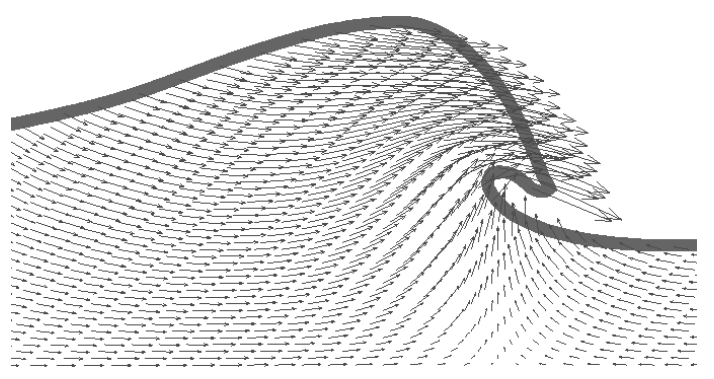

(a) Water

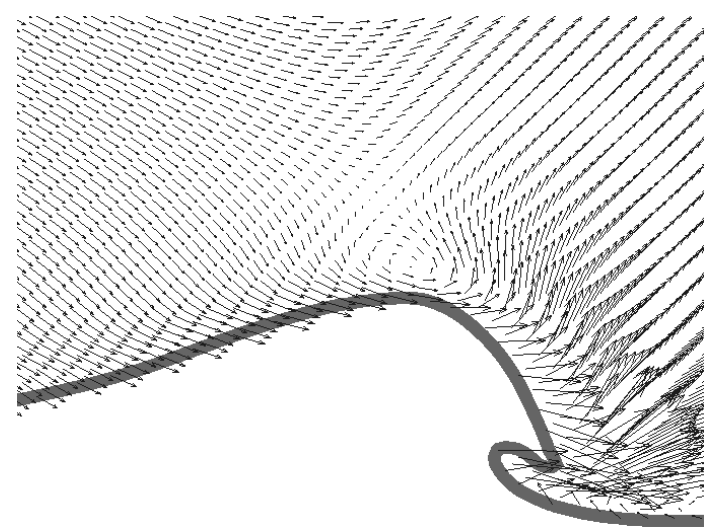

(b) Air

Fig. 9. Velocity field in both media. Only one vector over three is shown in each medium. Sections taken from a 3D simulation. $t=0.39 T s, C=0.5$. 


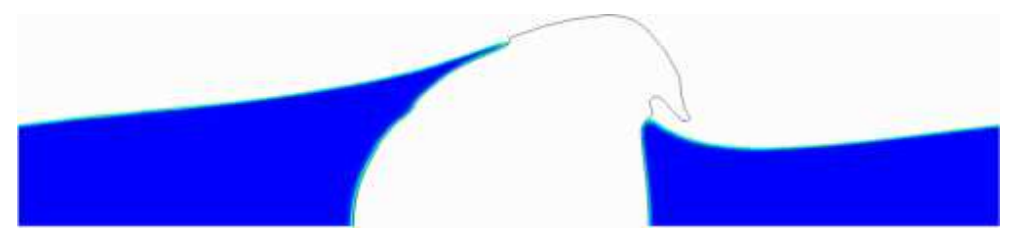

Fig. 10. Impacting jet. Sections taken from a 3D simulation. $\frac{H}{L}=0.13, \frac{d}{L}=0.13$, $C=0.5$.

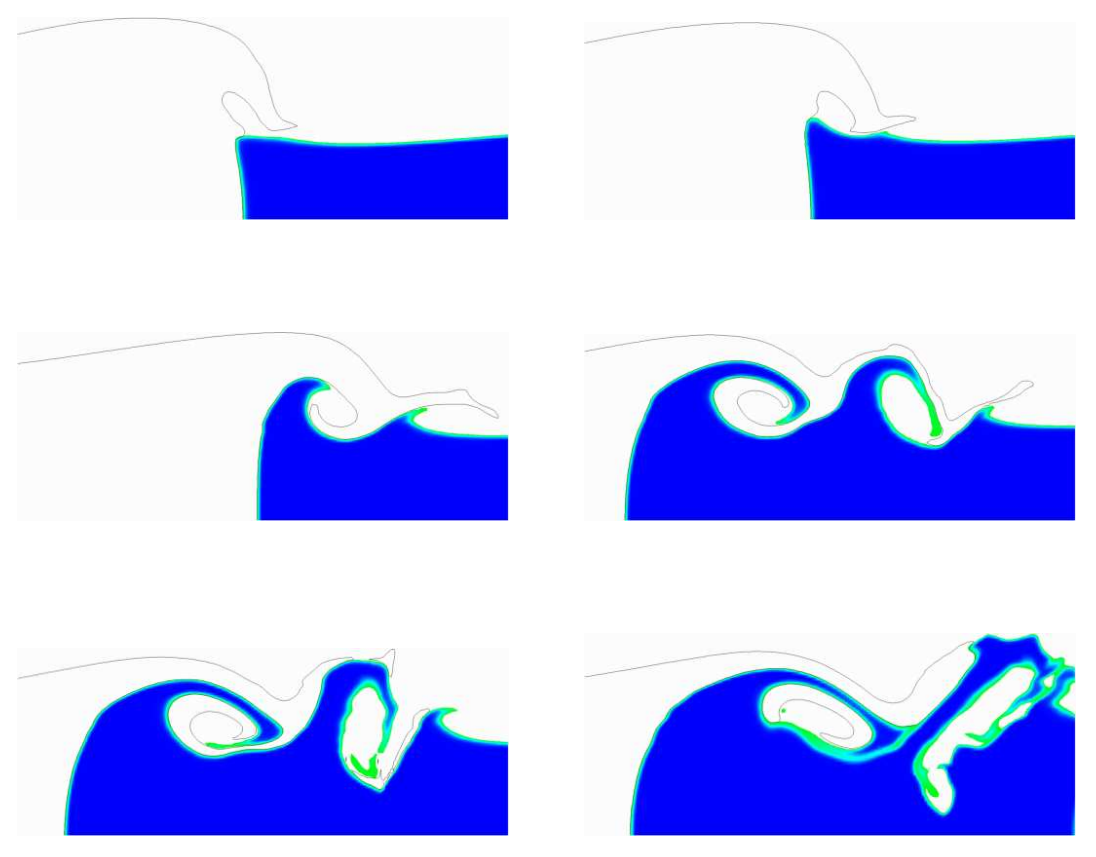

Fig. 11. Splash-up generation. Sections taken from a $3 \mathrm{D}$ simulation. $\frac{H}{L}=0.10$, $\frac{d}{L}=0.10, C \geq 0.5$. 

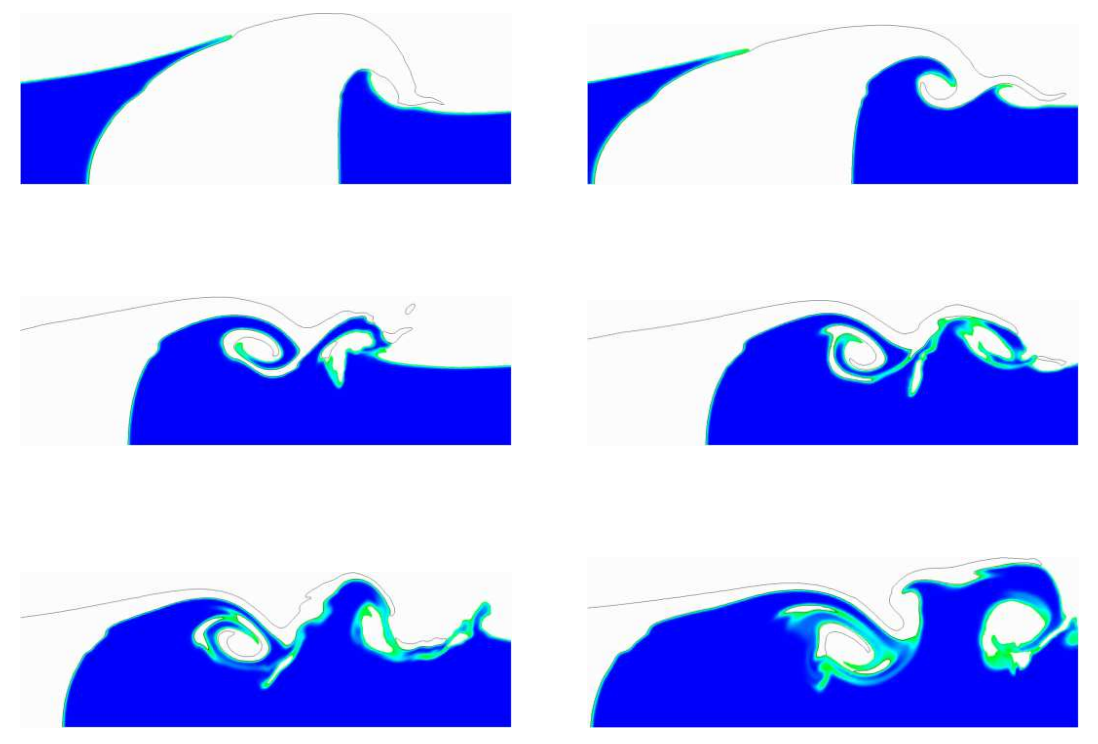

Fig. 12. Splash-up generation. Sections taken from a $3 \mathrm{D}$ simulation. $\frac{H}{L}=0.13$, $\frac{d}{L}=0.13, C \geq 0.5$.
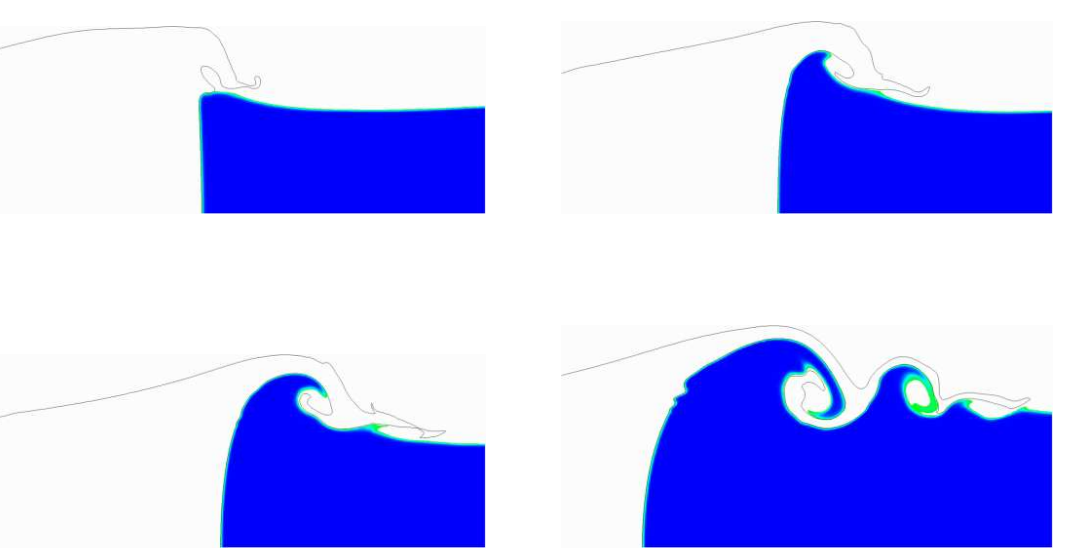

Fig. 13. Splash-up generation. Sections taken from a 3D simulation. $\frac{H}{L}=0.13$, $\frac{d}{L}=0.17, C \geq 0.5$. 


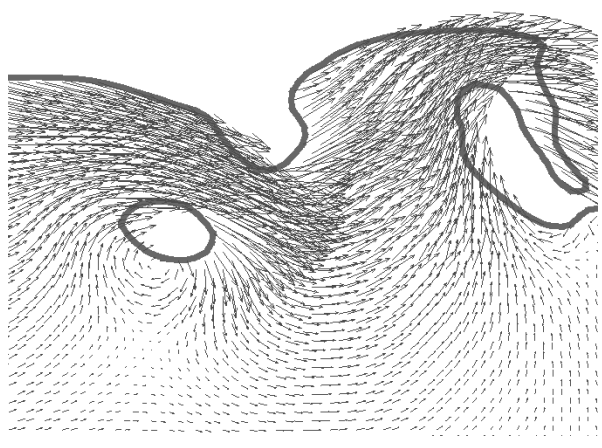

(a)

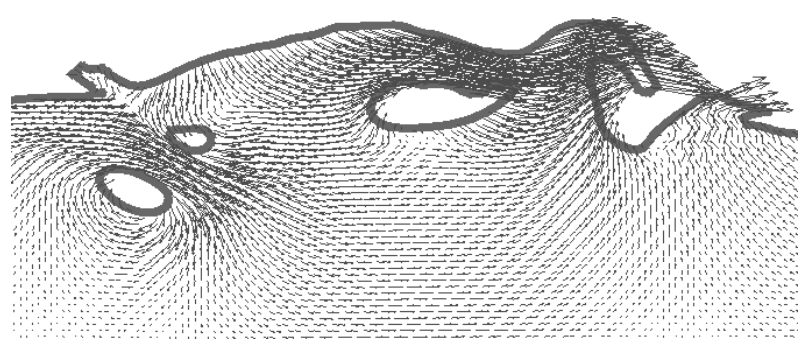

(b)

Fig. 14. Splash-up generation. Sections taken from a 3D simulation. Only one vector over three is shown in the water. $\frac{H}{L}=0.13, \frac{d}{L}=0.13, C=0.5$.

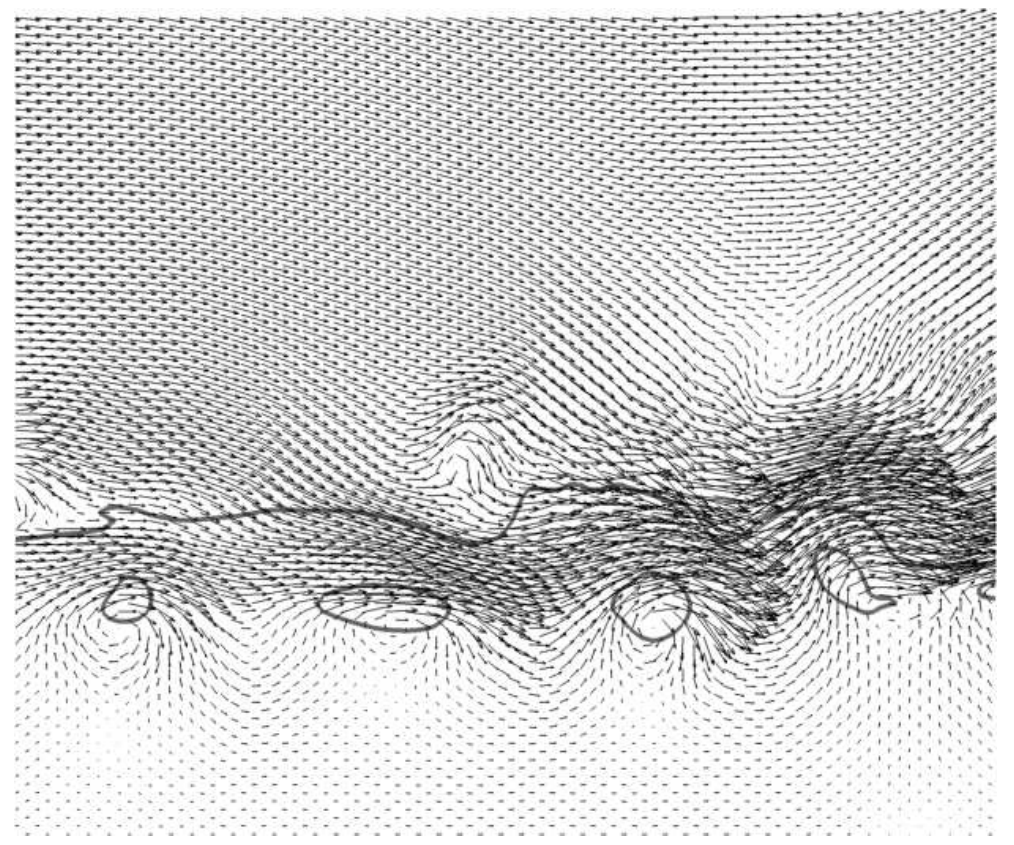

Fig. 15. Co-rotative vortices. Only one vector over two is shown in the water. $\frac{H}{L}=0.08, \frac{d}{L}=0.10, C>0.5$. 


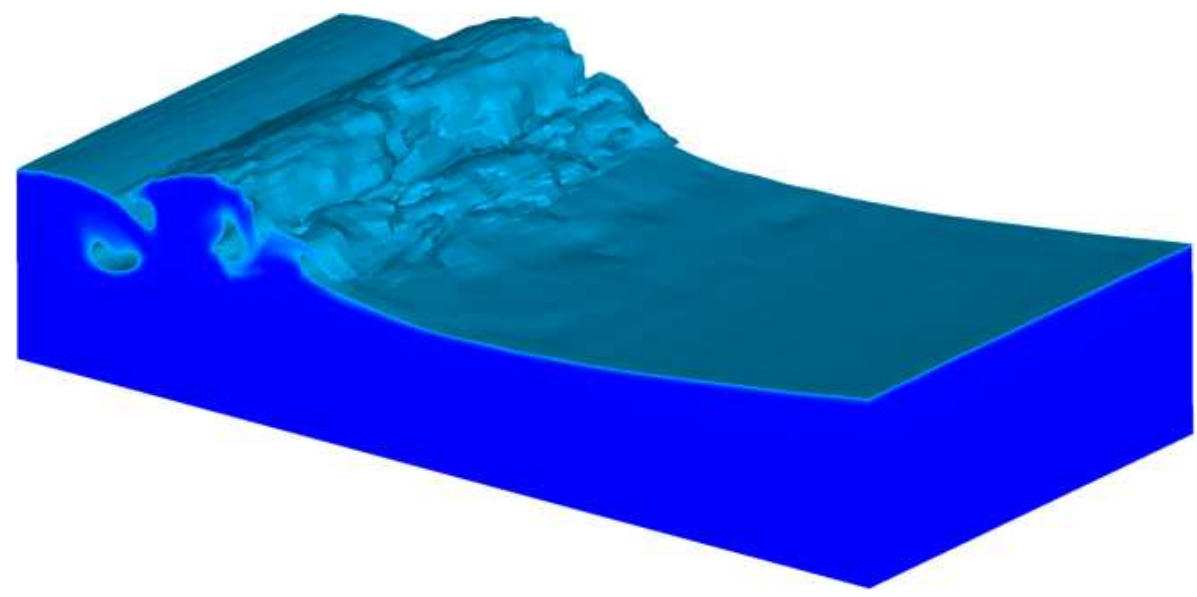

(a) $t=0.28 \mathrm{~s}$

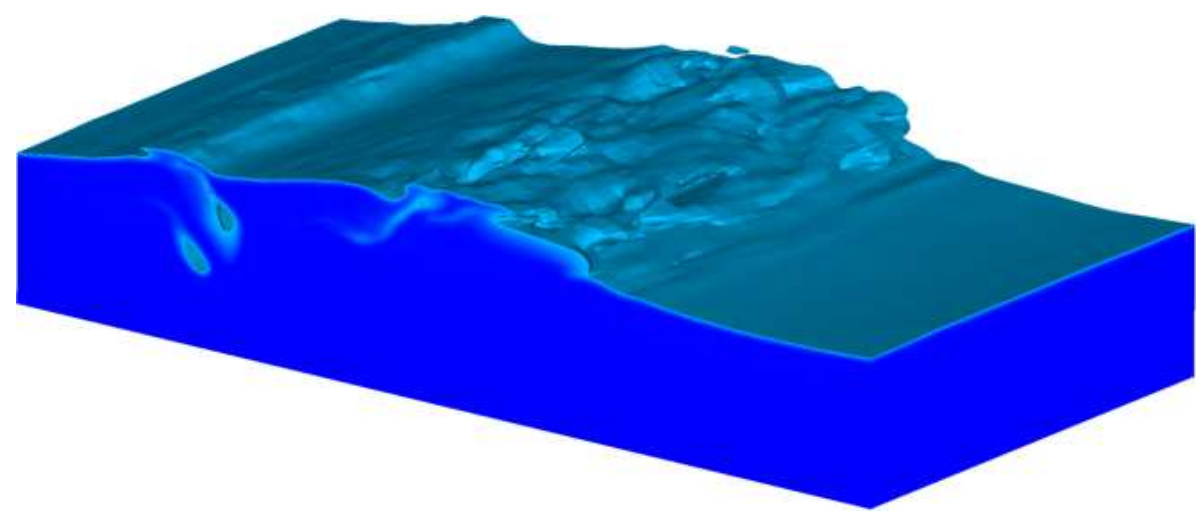

(b) $t=0.35 \mathrm{~s}$

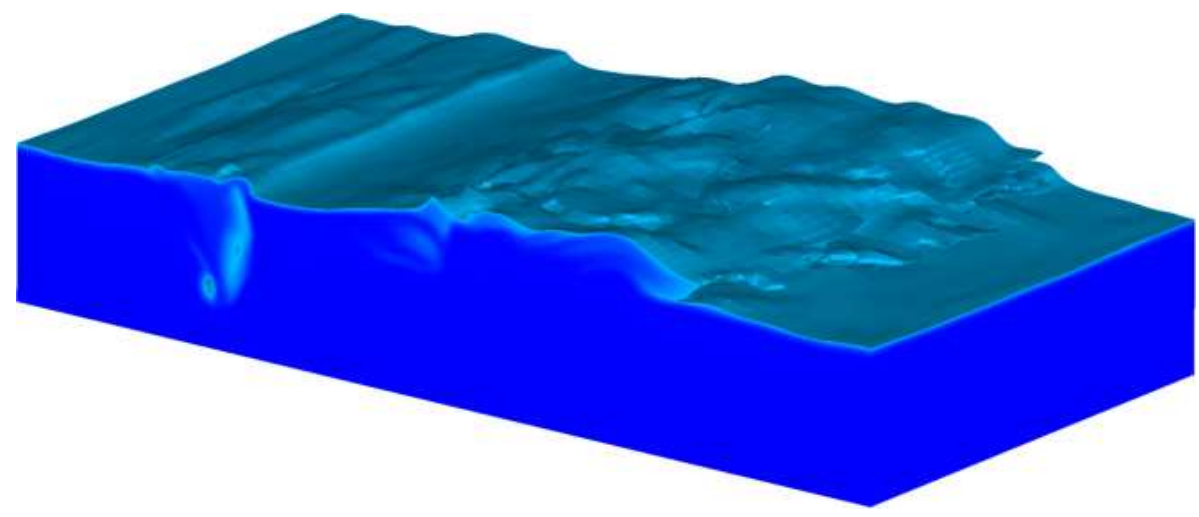

(c) $t=0.42 \mathrm{~s}$

Fig. 16. Three-dimensional large eddy simulation of a plunging breaker. $\frac{H}{L}=0.13$, $\frac{d}{L}=0.17, C \geq 0.5$. 


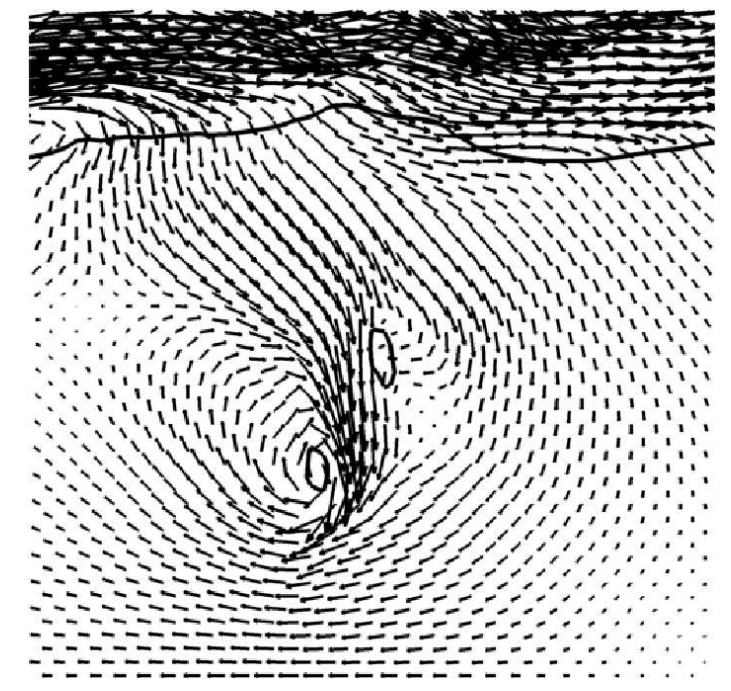

(a) Right side

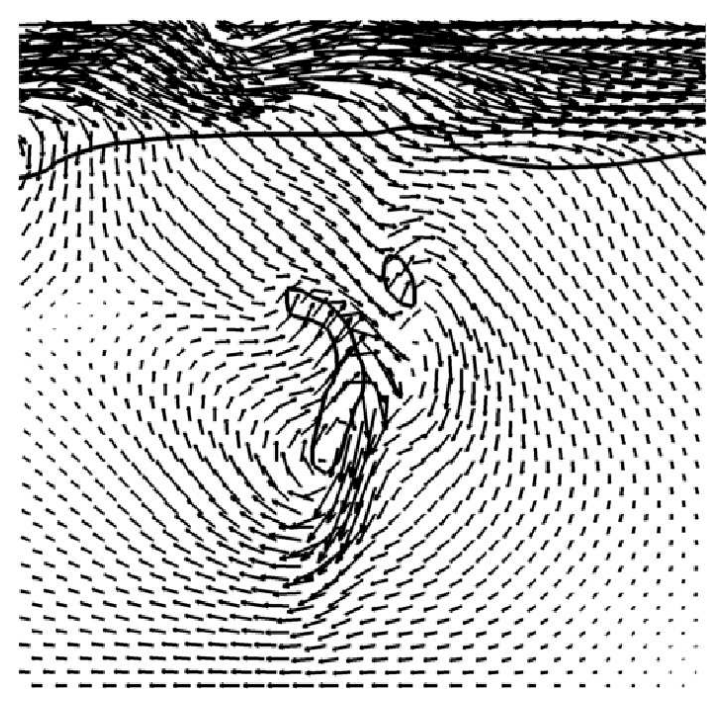

(b) Left side

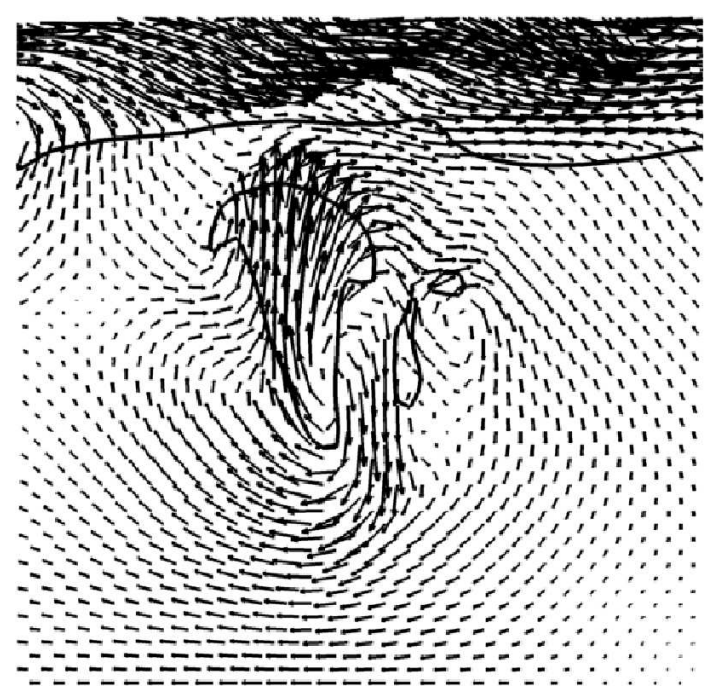

(c) Middle

Fig. 17. Velocity field in both air and water media showing the main gas pocket rising to the surface - zoom of vertical sections taken from the three-dimensional large eddy simulation of a plunging breaker, with only one vector over two. $\frac{H}{L}=0.13$, $\frac{d}{L}=0.17, C=0.5, t=0.40 \mathrm{~s}$. 


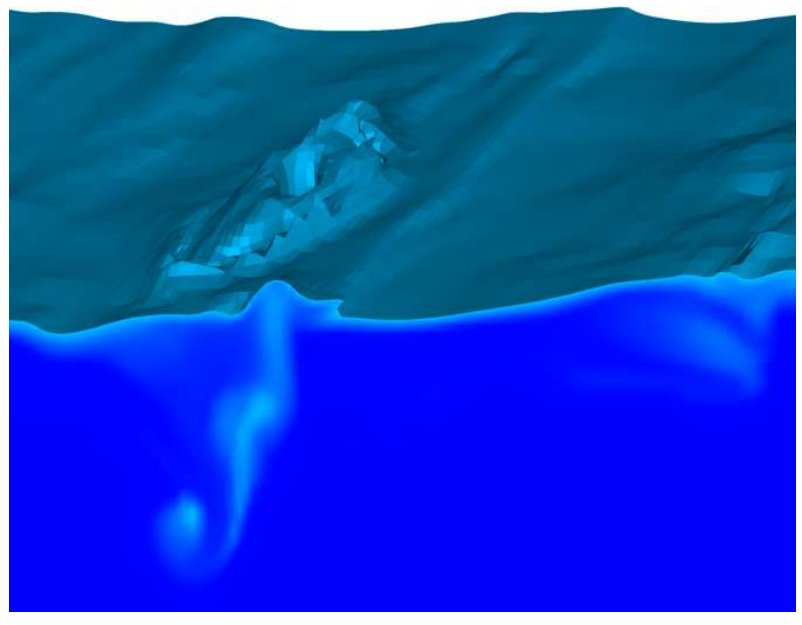

(a)

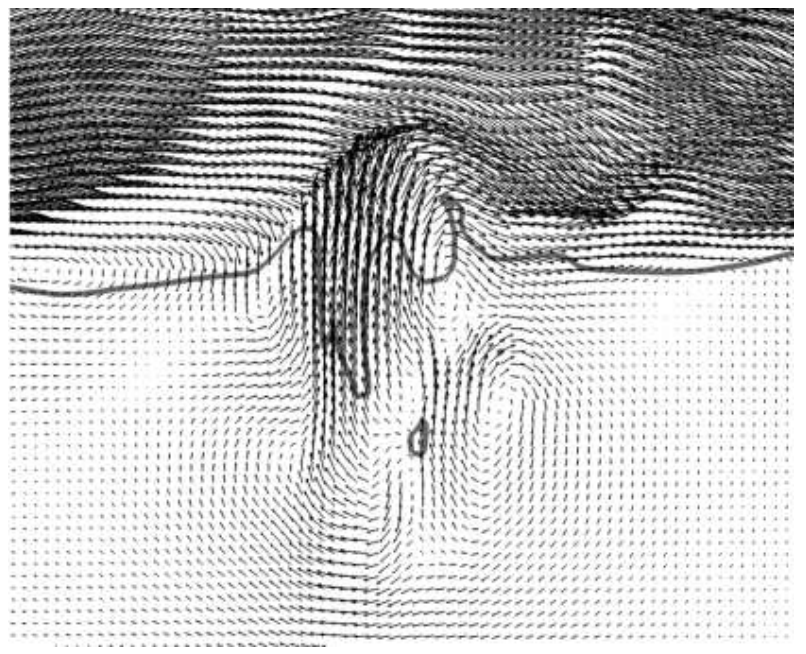

(b)

Fig. 18. (a) Main gas pocket exploding through the free surface - zoom of the three-dimensional numerical domain. (b) Velocity field in both air and water media showing the main gas pocket once it pierced the free surface - zoom of a vertical middle section taken from the three-dimensional large eddy simulation of a plunging breaker $\frac{H}{L}=0.13, \frac{d}{L}=0.17, C=0.5, t=0.43 s$. 


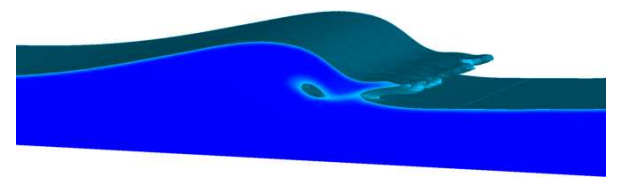

(a) $t=0.16 \mathrm{~s}$

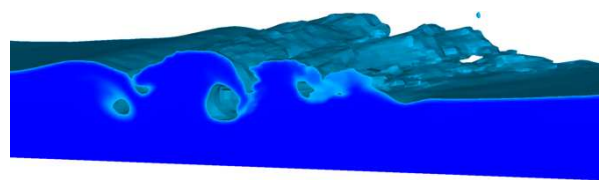

(c) $t=0.27 \mathrm{~s}$

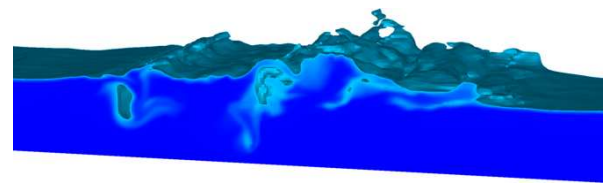

(e) $t=0.34 \mathrm{~s}$

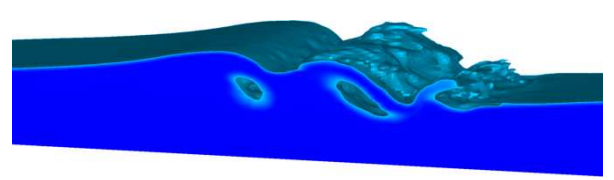

(b) $t=0.22 \mathrm{~s}$

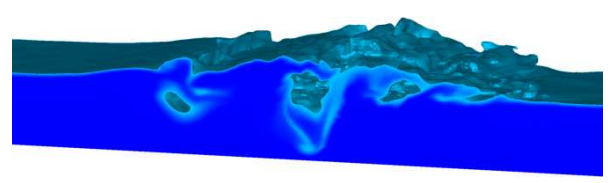

(d) $t=0.30 \mathrm{~s}$

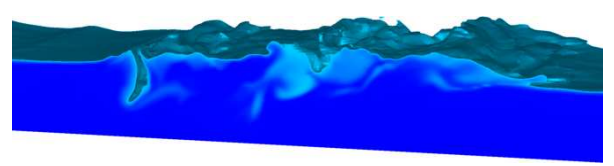

(f) $t=0.37 \mathrm{~s}$

Fig. 19. Snap shots of a three-dimensional large eddy simulation of a plunging breaker. $\frac{H}{L}=0.13, \frac{d}{L}=0.13, C \geq 0.5$. 


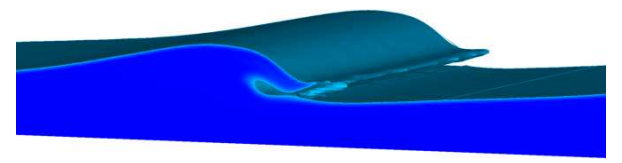

(a) $t=0.16 \mathrm{~s}$

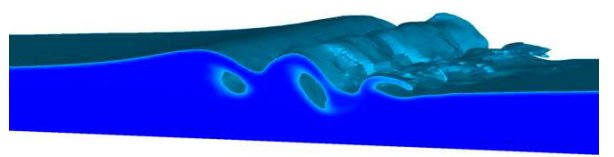

(c) $t=0.23 \mathrm{~s}$

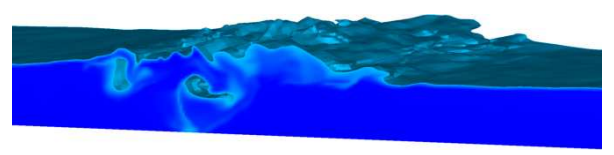

(e) $t=0.35 \mathrm{~s}$

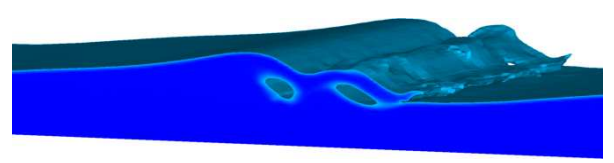

(b) $t=0.21 \mathrm{~s}$

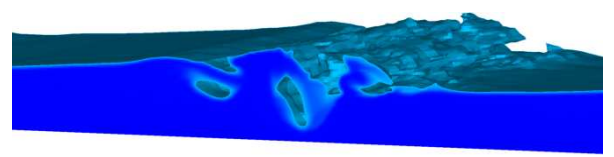

(d) $t=0.28 \mathrm{~s}$

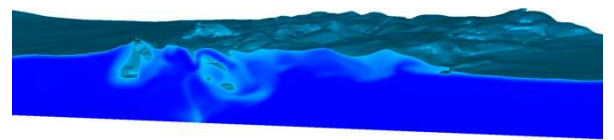

(f) $t=0.38 \mathrm{~s}$

Fig. 20. Snap shots of a three-dimensional large eddy simulation of a plunging breaker. $\frac{H}{L}=0.10, \frac{d}{L}=0.10, C \geq 0.5$. 


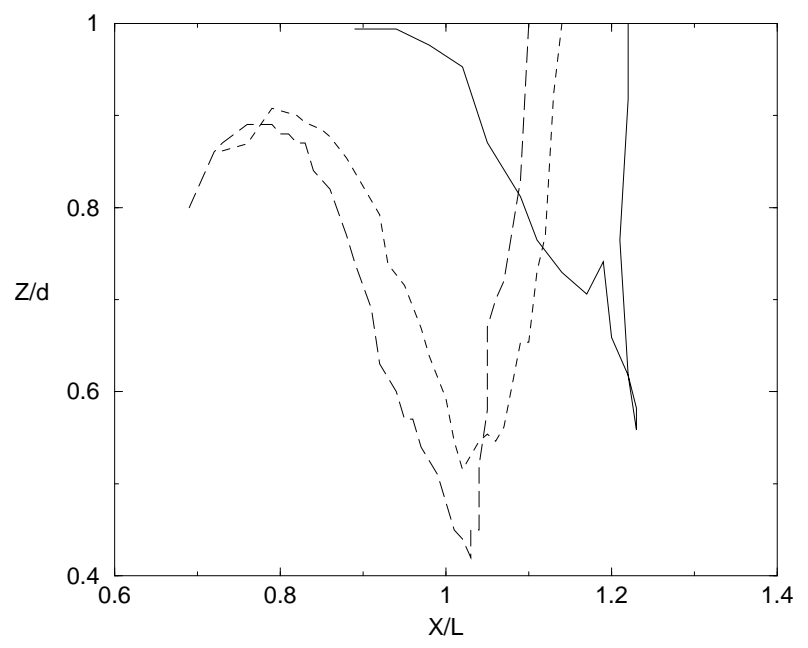

(a) Main pocket of air

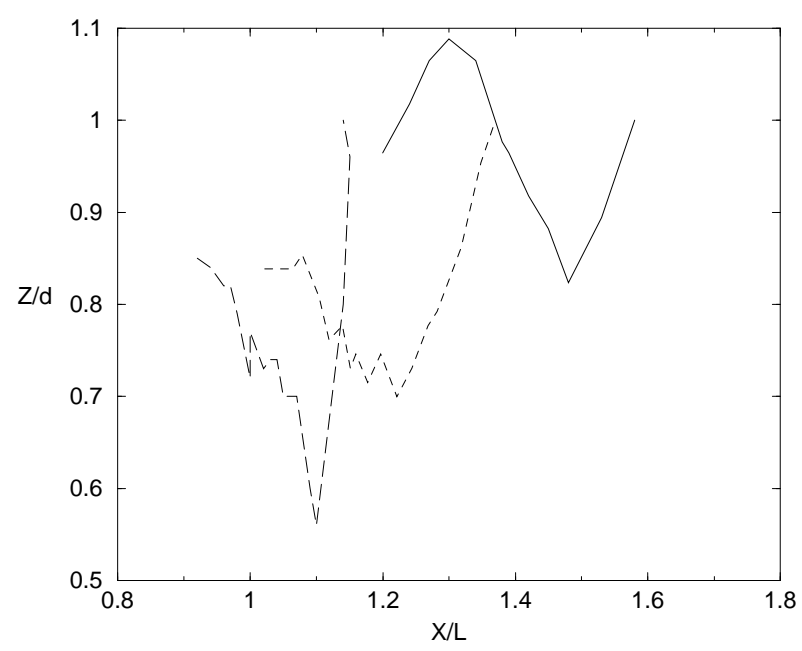

(b) First co-rotative spinning pocket of air

Fig. 21. Trajectories of the main pocket of air entrained in the water for each three-dimensional large eddy simulations. The abscissae are divided by the wavelength $L$ and the ordinates are divided by the initial water depth $d$. Solid line: $\frac{H}{L}=0.13$, $\frac{d}{L}=0.17$; long dashed line: $\frac{H}{L}=0.10, \frac{d}{L}=0.10$; dashed line: $\frac{H}{L}=0.13, \frac{d}{L}=0.13$. 


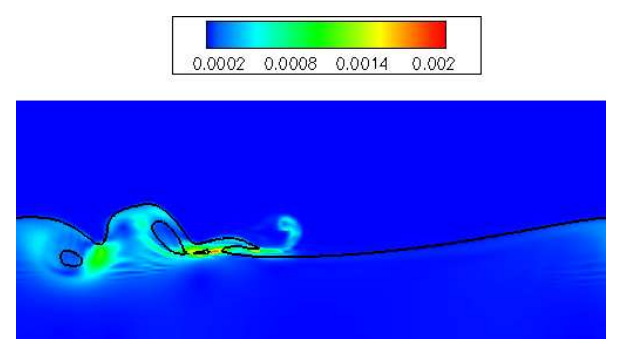

(a) $t=0.27 \mathrm{~s}$

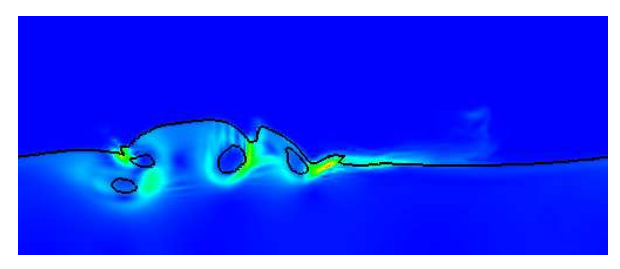

(c) $t=0.33 \mathrm{~s}$

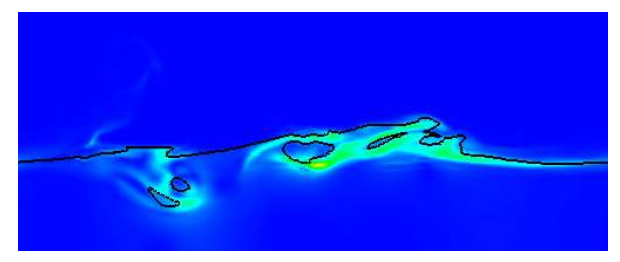

(e) $t=0.36 \mathrm{~s}$

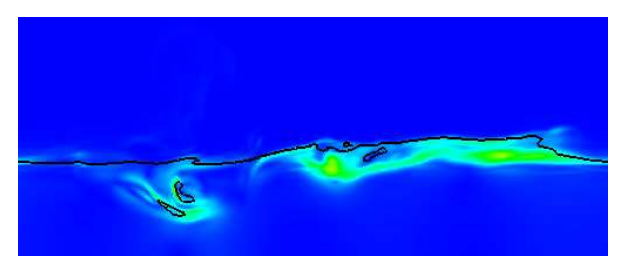

(g) $t=0.43 \mathrm{~s}$

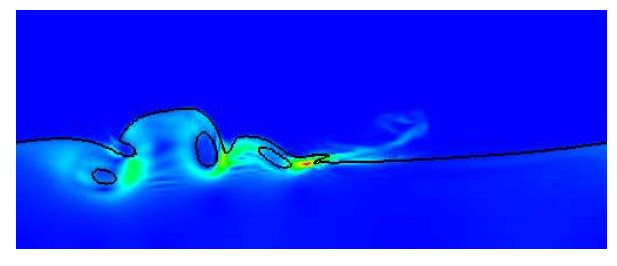

(b) $t=0.30 \mathrm{~s}$

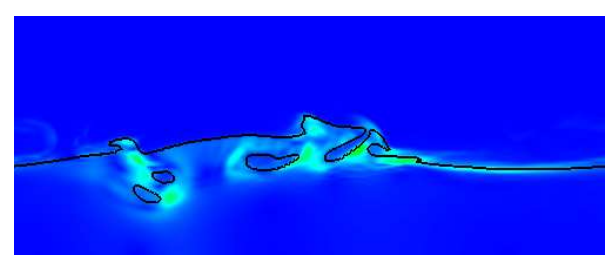

(d) $t=0.34 \mathrm{~s}$

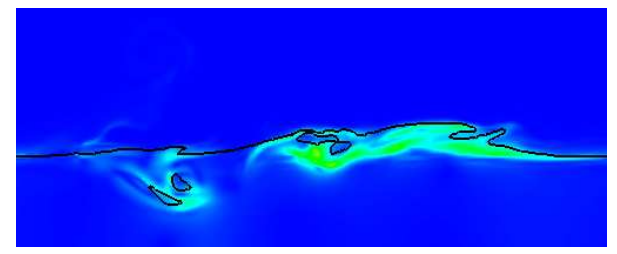

(f) $t=0.40 \mathrm{~s}$

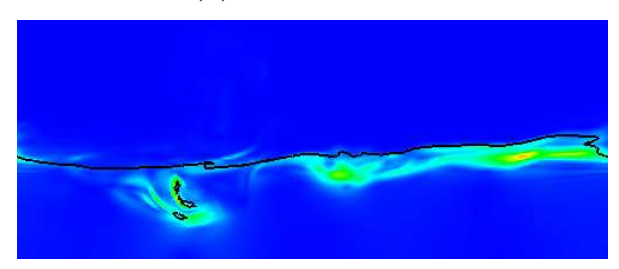

(h) $t=0.45 \mathrm{~s}$

Fig. 22. Turbulent viscosity magnitude $\left(\mathrm{m}^{2} . \mathrm{s}^{-1}\right)$ in both air and water media. The black line stands for $C=0.5$. Sections taken in the middle plane of a 3D simulation. $\frac{H}{L}=0.13, \frac{d}{L}=0.17$. 


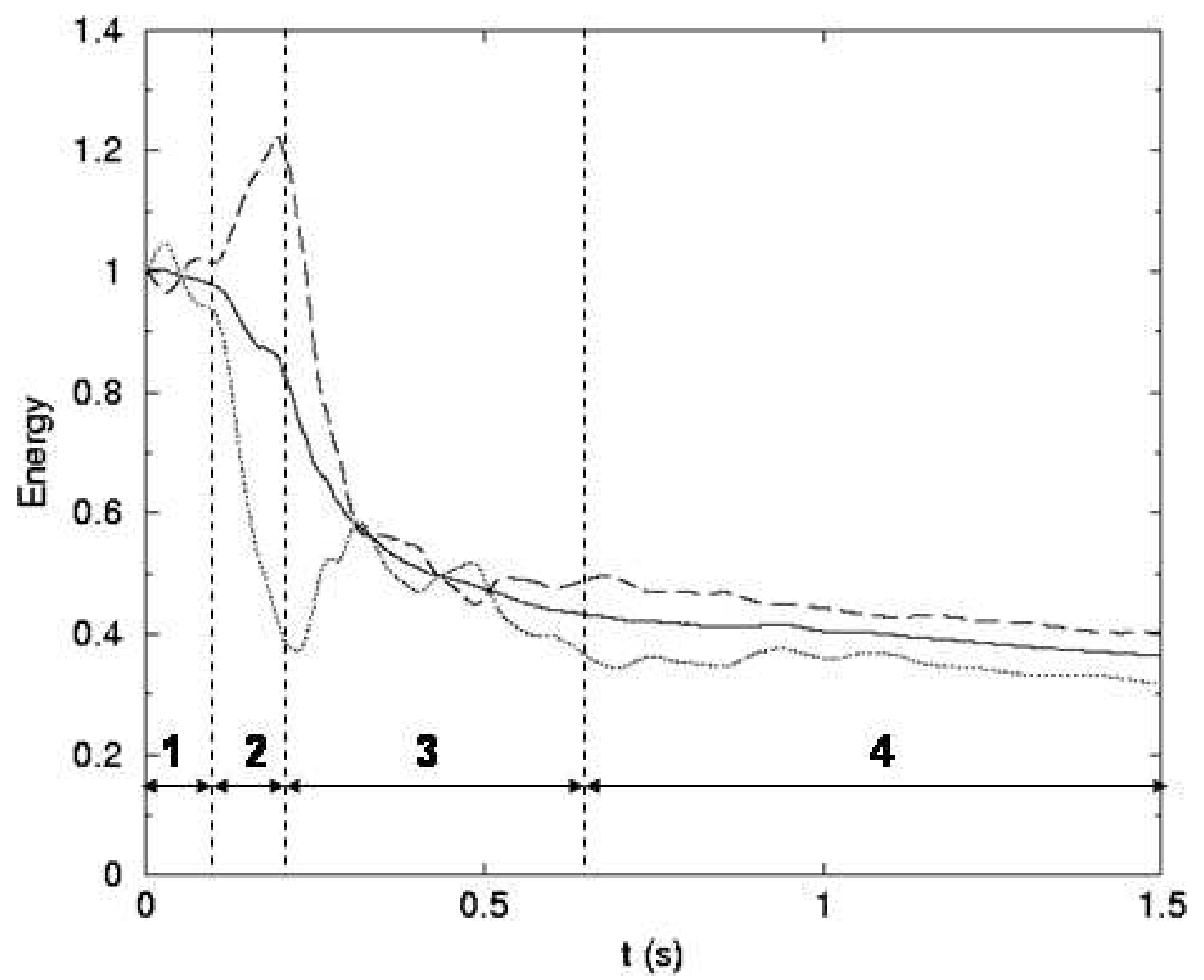

Fig. 23. Time evolution of the non-dimensional energies. Solid line: total energy; dashed line: kinetic energy; dotted line: potential energy. $\frac{H}{L}=0.10, \frac{d}{L}=0.10$. Zone 1: steepening of the wave; zone 2 : jet ejection; zone 3 : jet impact and splash-ups generation; zone 4: residual flow. 


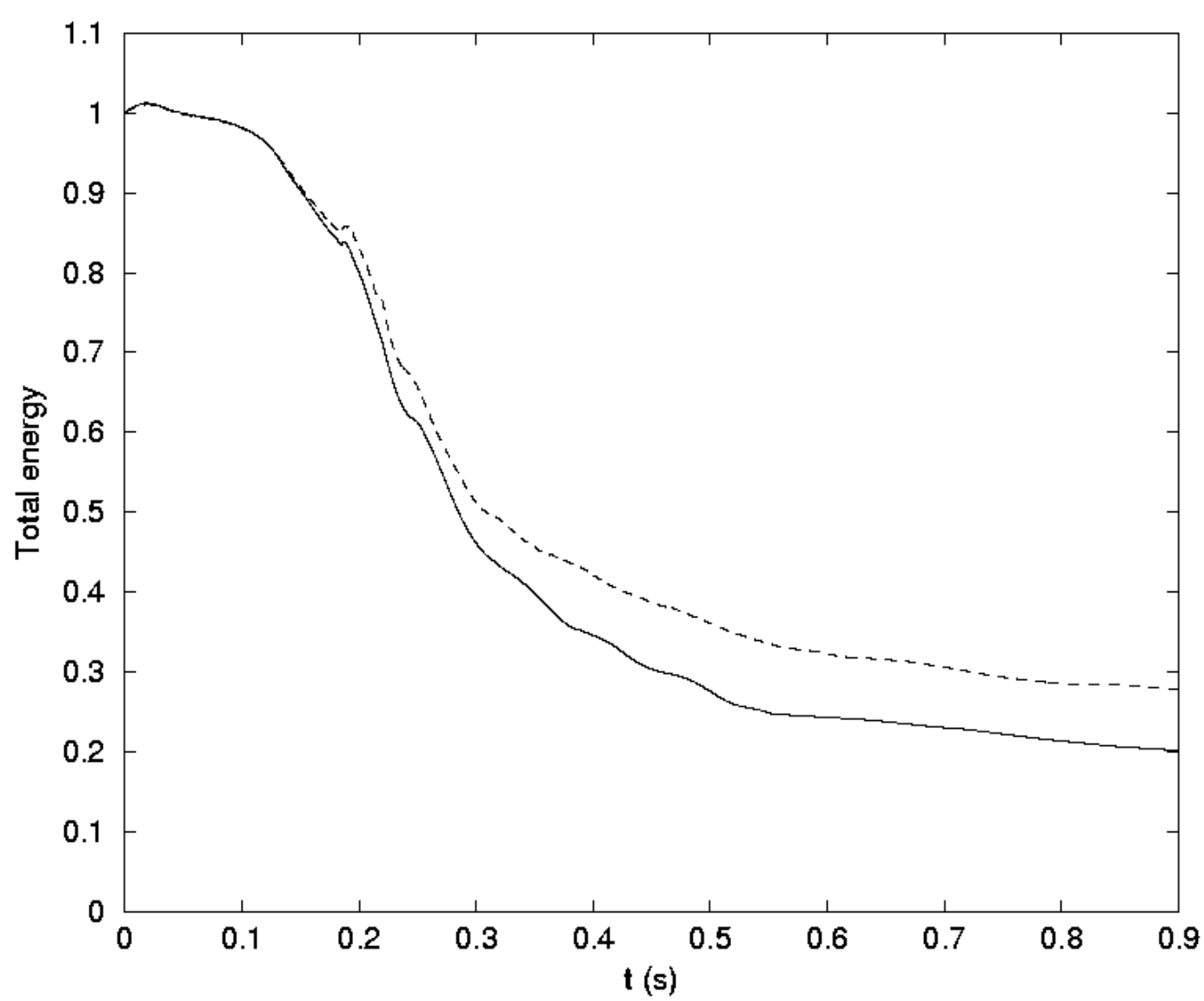

Fig. 24. Time evolution of the non-dimensional energy. Solid line: total energy calculated from a 3D simulation; dashed line: total energy calculated from a $2 \mathrm{D}$ simulation. $\frac{H}{L}=0.10, \frac{d}{L}=0.10$. 